\title{
Hodgkin Lymphoma in Children and Adolescents: Advances in Pathology, Diagnosis, and Treatment Strategies
}

\begin{abstract}
Strategies used for the treatment of children with Hodgkin lymphoma (HL) over the last four decades have resulted in excellent long-term survivals. However, the short- and long-term treatment-associated morbidities were high. In an attempt to reduce complications, the current treatment strategies apply initial risk stratification principles based on certain host and tumor risk factors to help assign patients to the appropriate risk group and tailor therapy based on response to chemotherapy (CTR). Radiotherapy (RT) was only given to certain groups of patients who show suboptimal response to CTR. Enrollment of patients in clinical trials allowed testing newer treatment strategies, which has improved the outcome significantly. High-dose CTR with stem cell support remains the mainstay of treatment for relapsed and refractory HL. Newer targeted medications are being increasingly used for the treatment of patients with relapsed and refractory HL, but results are variable. In this review report, we give extensive account about the pathophysiology, clinical presentation, histopathological diagnosis, modern investigation techniques, the most recent risk adapted treatment strategies, and the use and effect of novel medications. In addition, we discuss in details the short- and long-term therapy-related complications and future prospects in the management of HL.
\end{abstract}

Keywords: ${ }^{18}$ Fluorodeoxyglucose-positron emission tomography scan, chemotherapy, Hodgkin lymphoma, pathological classification, radiotherapy, staging system

\section{Introduction}

Hodgkin lymphoma (HL) affects nearly 7000 adult and pediatric patients every year in the United States of America (USA). With the currently available therapies, nearly $89 \%$ of them will be cured. There were 458 new cases of pediatric and adolescent HL patients diagnosed in the USA in the year 2017, accounting for nearly $3 \%$ of all pediatric cancers..$^{[1,2]}$

According to the Surveillance, Epidemiology, and End Results (SEER) program of the National Cancer Institute, USA, HL had a bimodal distribution of age-specific incidence rates with two peaks at the 15-34 years and the older than 55 years age groups. Since 1973, the incidence of HL in the younger age group increased progressively mainly as a result of a marked increase in the incidence of the nodular sclerosis (NS) histological subtype. The increase in incidence over time was much greater in young adult women. The overall incidence

This is an open access journal, and articles are distributed under the terms of the Creative Commons Attribution-NonCommercial-ShareAlike 4.0 License, which allows others to remix, tweak, and build upon the work non-commercially, as long as appropriate credit is given and the new creations are licensed under the identical terms.

For reprints contact: WKHLRPMedknow_reprints@wolterskluwer.com of the mixed cellularity (MC) and the lymphocytic predominance (LP) subtype (23.4\% and $6 \%$, respectively), remained stable. The incidence of the lymphocytic depletion (LD) subtype (3.8\%), occurred predominantly in the elderly, has also decreased. Similarly, unclassifiable cases of HL designated as miscellaneous $(9.1 \%)$ decreased over time, probably as a result of improved classification. Nevertheless, a subset of cases of HL remained difficult to subclassify. The human immunodeficiency virus (HIV) epidemic appears to be associated with an increased incidence of HL in adult males aged 30-49 years. ${ }^{[2,3]}$

Childhood HL is not a biologically distinct disease. It differs from adult HL primarily in the incidence of disease histological type. Preadolescent children are more likely predominant HL Adolescent and young adult $\mathrm{HL}$ are indistinguishable, with the NS subtype predominating. ${ }^{[4]}$

With few exceptions, prior to the year 1960, HL was almost universally fatal

\footnotetext{
How to cite this article: Abbas AA, Almaghraby HQ. Hodgkin lymphoma in children and adolescents: Advances in pathology, diagnosis, and treatment strategies. Indian J Med Paediatr Oncol 2020;41:492-509.
} to develop MC and nodular lymphocyte

\section{Adil Abdelhameed \\ Abbas ${ }^{1,2}$, Hatim Qasim Almaghraby ${ }^{3,4}$}

${ }^{1}$ Department of Pediatrics, College of Medicine, King Saud Bin Abdulaziz University for Health Sciences, Riyadh, Saudi Arabia, ${ }^{2}$ Princess Nourah Oncology Centre, King Abdulaziz Medical City, Jeddah, Saudi Arabia, ${ }^{3}$ Department of Pathology, College of Medicine, King Saud Bin Abdulaziz University for Health Sciences, Riyadh, Saudi Arabia, ${ }^{4}$ Department of Laboratory Medicine, King Abdulaziz Medical City, Jeddah, Saudi Arabia

Submitted: 05-Apr-2020 Accepted in Revised Form: 18-Apr-2020

Published: 29-Aug-2020

\section{Address for correspondence:} Dr. Adil Abdelhameed Abbas, Department of Pediatrics, King Saud Bin Abdulaziz University for Health Sciences, Riyadh, Kingdom of Saudi Arabia. Princess Nourah Oncology Centre, King Abdulaziz Medical City, P. O. Box: 9515, Jeddah 21423, Kingdom of Saudi Arabia. E-mail: adilahabbas2000@yahoo.com. au

Access this article online Website: www.ijmpo.org

DOI: 10.4103/ijmpo.ijmpo_135_20 Quick Response Code:

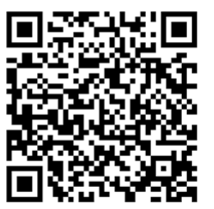


disease adult and pediatric patients. Since that time, it was very clear that HL is a systemic cancer and needs systemic treatment rather than local control as many cases of apparently localized disease treated with surgical resection were soon followed by local and metastatic relapse. Long-term survivals were only seen after the start of multi-model chemotherapy-radiotherapy (CTR-RT) in the early 1970s, and that coincided with the development of new methods to establish the correct diagnosis and define the exact clinical disease stage. Patient's enrollment in clinical trials gradually defined appropriate treatment strategies depending on certain pretreatment risk factors. Earlier clinical trials tested the therapeutic effects of RT or CTR, then tested combination chemo-RT for the different clinical stages using favorable versus unfavorable risk factors. The standard treatment for advanced stage or unfavorable disease is 4-6 cycles of intense multiagent noncross-resistant CTR in addition to involved-field radiotherapy (IFRT). Response-adapted therapy has recently emerged as a promising strategy to tailor treatment intensity and thereby reduce toxicity in children with an excellent prognosis and use more intensive therapy in those at higher risk of progression or relapse. ${ }^{[5]}$ Tremendous success has been achieved with the use of combined modality therapy (CMT) with the overall survival (OS) and event-free survival (EFS), reaching high figures, however, this achievement came on the expense of increasing rate of serious long-term side effects. To reduce the side effects of therapy while maintaining the same or even further improving survival figures, the modern therapeutic approach involves the concepts of risk stratification and response-based treatment. Strict clinical and radiological criteria are being applied to determine the patient risk group and subsequently place him/her on the right treatment strategy. Response assessment, likewise, is carried out using similar preset strict radiological criteria. Computed tomography (CT) and magnetic resonance image (MRI) scans remain the preferred radiological tests to determine tissue involvement. CT scan examination has now totally replaced the need for the previously used invasive procedures such as exploratory laparotomy and splenectomy. ${ }^{[6]}$

\section{The World Health Organization Histopathological Classification of Hodgkin Lymphoma}

The current World Health Organization classification of HL generally distinguishes the relatively rare variant of nodular lymphocyte-predominant Hodgkin lymphoma (NLPHL) subtype from a second group, which comprises the classical HL. According to the classification, classical HL is further separated into four subtypes; lymphocyte-rich (LR), NS, MC, and LD subtypes. The distinction between classical HL subtypes is based on the typical morphology in addition to the immunohistochemical characteristics of the Hodgkin and Reed-Sternberg cells (HRS) (CD30+, CD15+, and CD20-). The NLPHL type has similarities with non-Hodgkin lymphoma (NHL) in many aspects [Figure 1a-i]. On the molecular biology level, the tumor cells of all kinds of HL are clonal B cells derived from germinal center cells. However, tumor cells of NLPHL differ from classical HL by patterns of somatic mutations. Due to inability of HRS cells to express a B cell receptor, they should perish under normal conditions. However, they escape from apoptosis by novel mechanisms which are only partially understood, such as genomic mutations of the 1-kappa B gene and the fas receptor gene and downregulation of B cell markers. In rare cases, HRS cells of HL can be derived from T cells, as could be demonstrated by single-cell analysis. These results suggest that future classifications of HL will not only take into account morphological and phenotypical profile, but also genetic profile and mechanisms of transformation. ${ }^{[7,8]}$

\section{Role of Epstein-Barr Virus in the Pathogenesis of Hodgkin Lymphoma}

Epstein-Barr virus (EBV) is detected in nearly $70 \%$ of classical HL neoplastic cells. NLPHL is generally considered EBV negative. ${ }^{[9]}$ EBV genomes and deoxy-riboneocleic acid (DNA) products can be detected in HRS cells using polymerase chain reaction (PCR). The prevalence of EBV-associated HL in any population group varies according to age, sex, ethnicity, and histologic subtype. Viruses such as the EBV and HIV are thought to be involved in B cell-derived HL pathogenesis. ${ }^{[10]}$ Two epidemiologic studies have examined risk factor profiles in HL with taking into account the EBV status. There is a small peak in incidence in young adults (15-24 years) and a second larger peak in older adults for EBV-associated HL cases. By contrast, EBV-negative HL accounts for the major part of the peak incidence observed in young adults, after which the incidence of this entity declines. Previous infectious mononucleosis is associated with an increased risk of HL. EBV-associated HL in childhood and young adults, therefore, appears to follow previous infection by EBV virus. EBV-associated HL patients have an increased frequency of circulating EBV-infected cells at the time of diagnosis compared to patients with EBV-negative HL patients and normal controls. In EBV-associated HL cases, EBV genomes are detectable in the serum and plasma, and EBV genome copy number may provide an indication of tumor burden and prove to be a useful marker for disease monitoring. The etiology of EBV-negative HL, however, remains largely unknown. While the involvement of an infectious agent in the pathogenesis is suspected, none has yet been identified. ${ }^{[11]}$

The prognostic significance of EBV infection in $\mathrm{HL}$ is not known, however increased pretreatment levels of plasma EBV DNA determined PCR, are associated with lower survival especially in patients with previously untreated, advanced-stage HL. ${ }^{[12,13]}$ 


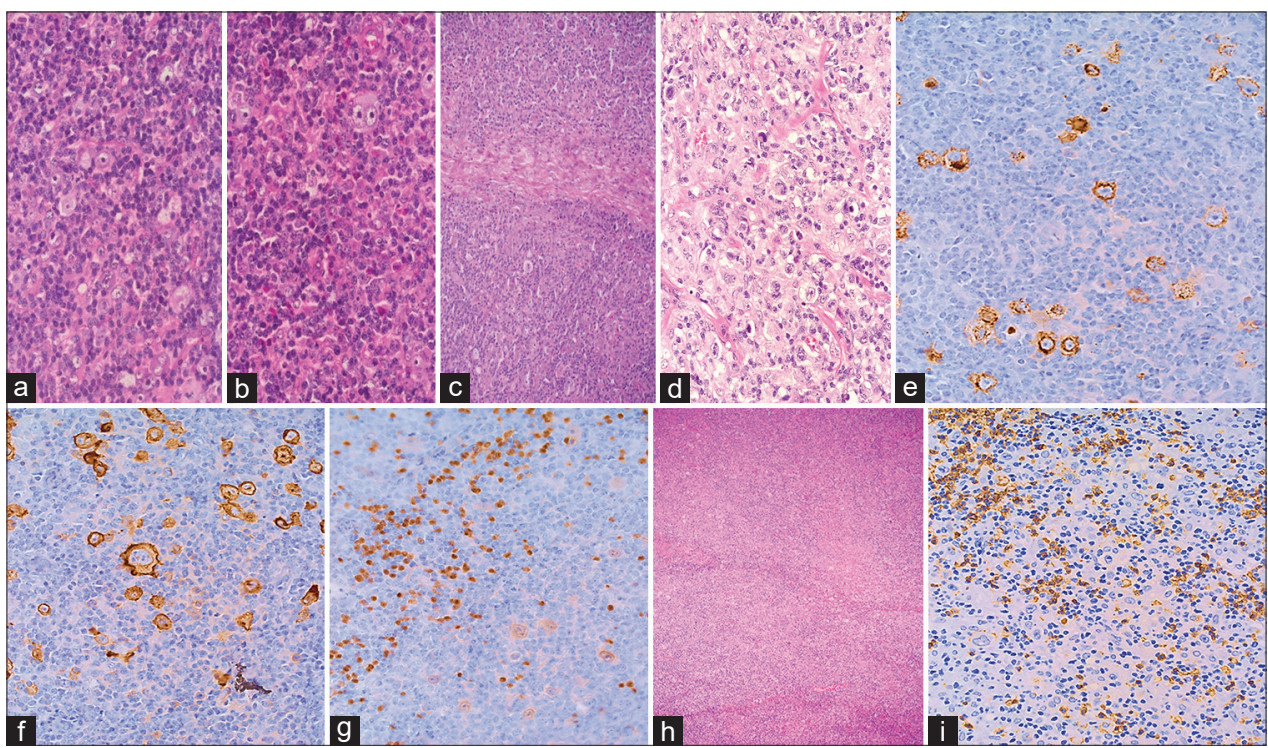

Figure 1: (a) Classical Hodgkin lymphoma, lymphocyte-rich subtype. Scattered classical large binucleated cells. The nuclei are large and are surrounded by vesicular chromatin and prominent large nucleoli. The background shows few variant Hodgkin-Reed-Sternberg cells and infiltration with predominant T-lymphocytes, plasma cells, and immunoblasts ( $\mathrm{H}$ and E, $\times 200$ ). (b) Classical Hodgkin lymphoma, mixed cellularity subtype. Scattered classical large binucleated cells. The nuclei are large and are surrounded by vesicular chromatin and prominent large nucleoli. The background shows few variant Hodgkin and Reed-Sternberg cells and mixed inflammatory infiltrates comprised T-lymphocytes, eosinophils, plasma cells, and macrophages with few less developed fibrous bands crossing in between but no capsular fibrosis $(H \times E, \times 200)$. (c) Classical Hodgkin lymphoma, nodular sclerosis subtype. Thick fibrous bands and pericapsular fibrosis surrounding nodules of tumor composed of Hodgkin and reed-sternberg cells and a background mixed lymphoid and macrophage inflammatory cells $(H$ and $E, \times 200)$. (d) Classical Hodgkin lymphoma, lymphocytic depletion subtype. More frequent Hodgkin and reed-sternberg cells are seen in a background of few scattered lymphocytic inflammatory cells $(H$ and $E, \times 200)$. (e) Immunohistochemistry: Classical Hodgkin lymphoma: CD15, a cell surface carbohydrate antigen, a marker that characteristically positively stain Hodgkin and reed-sternberg cells brown in a membranous and golgi patterns of staining (Ventana, Carpentaria, CA, ×200). (f) Immunohistochemistry: Classical Hodgkin lymphoma: CD30, a protein related to tissue necrotic factor- receptor family, characteristically positively stain Hodgkin and reed-sternberg cells brown in a membranous and golgi patterns (Ventana, Carpentaria, CA, ×200). (g) Immunohistochemistry: PAX5, a paired-box transcription factor of early B-lymphocyte development, weakly stains classical Hodgkin and reed-sternberg cells brown (Ventana, Carpentaria, CA, ×200). (h) Nodular lymphocyte-predominant Hodgkin lymphoma: In this lymphohistiocytic type of nodular lymphocyte-predominant Hodgkin lymphoma (LP in inset), scattered cells similar in-size to classical Hodgkin and reed-sternberg are seen (LP cells), however, these cells show large nuclei with single folded nuclear membrane. There are multiple but small size basophilic nucleoli. The cells lie on a non - neoplastic background mostly consist of B lymphocytes, plasma cells, and histiocytes. (i) Immunohistochemistry: CD20, typically positively stains LP cells of nodular lymphocyte-predominant Hodgkin lymphoma brown. LP cells are usually negative for CD30 and CD15 immune stains. Classical Hodgkin lymphoma, Hodgkin and reed-sternberg cells are typically negative for CD20 immunostaining

\section{Clinical Presentation}

Progressive painless enlargement of peripheral lymph nodes (LN) is the most common presentation of HL. Patients often present with persistent painless enlarged cervical or supraclavicular LNs, [Figure 2a and b]. LNs are typically rubbery or firm and are not tender to touch. With disease progression, the enlarged LNs form large nodal aggregates that may often become matted together and strongly attached to the underlying soft tissues. Differential diagnosis includes reactive lymphadenopathy and infection. Biopsy should be considered early when malignant disease is suspected. The presence of enlarged supraclavicular LNs should prompt earlier consideration of malignant disease as opposed to isolated cervical LN enlargement. At diagnosis, more than $70 \%$ of the cases involve cervical or supraclavicular LN enlargement. Cervical disease is accompanied with mediastinal LN enlargement in approximately two-thirds of the cases. Uncommonly, patients may present with isolated enlarged axillary or inguinal LNs. HL limited to infra-diaphragmatic site is rare and occurs in $<5 \%$ of pediatric patients. The liver and lung are the most common extranodal sites of spread followed by the bones. ${ }^{[14,15]}$ The clinical presentation of the disease in most patients is insidious with minimal symptoms. Less commonly, the disease may only be discovered incidentally, such as the discovery of large mediastinal mass in a patient undergoing radiologic examination for chronic cough or obstructive jaundice. Rarely, patients may present with acute medical emergency due to enlarged lymphatic tissue causing spinal cord compression or airway obstruction. Patients with bone marrow (BM) infiltration may rarely present with cytopenia. Except for the clinical stage, bulky disease, and the presence or absence of B symptoms, the prognostic significance of the different clinical presentations is not certain. ${ }^{[16]}$

\section{Extralymphatic Site Involvement}

\section{Liver involvement}

Primary hepatic HL is very rare and represents about $0.016 \%$ of all lymphomas. HL reaches the liver via hematogenous spread, and liver involvement is usually associated with widespread metastasis. Pattern of involvement varies between nodular lesions and diffuse hepatic infiltration. ${ }^{[17]}$ Prognosis and proper treatment in 
HL are strictly related to staging accuracy, and liver and spleen involvement is of great importance as they indicate advanced stage. The hepatic histopathology was studied by Dich et al., in 125 patients with HL to determine whether there are any features other than HRS cells that might aid in the diagnosis of hepatic involvement. Liver biopsy specimens from 41 patients with HL in the liver were compared with biopsy specimens from 84 patients without hepatic involvement. Patients with hepatic involvement by HL were much more likely to have histologic evidence of portal infiltrates larger than $1 \mathrm{~mm}$ in diameter $(78 \%$ vs. $1 \%)$, acute cholangitis ( $85 \%$ vs. $4 \%)$, portal edema $(90 \%$ vs. $8 \%$ ), and portal infiltrates with a predominance of atypical lymphocytes (78\% vs. $12 \%$ ) than patients with HL without hepatic involvement. The authors indicated that when these features are observed alone or in combination in a liver biopsy specimen from a patient with proven or suspected HL, careful search for HRS cells should be carried out. ${ }^{[17-19]}$

\section{Lung involvement}

Primary pulmonary lymphoma (PPL) is rare and represents $<1 \%$ of primary lung cancers. PPL represents approximately $10 \%$ of extranodal lymphoma. The majority of cases are NHLs, and primary pulmonary HL is very uncommon. Lung involvement with $\mathrm{HL}$ is often secondary to hematogenous spread or direct extension from hilar or mediastinal HL. The diagnosis is made if nodal involvement is absent, and the presence of disease elsewhere has been ruled out. Open lung biopsy is required to detect the unique pulmonary histopathologic changes. ${ }^{[20]}$ Kolygin and Vesnin studied 160 children with HL. Lung involvement was diagnosed in $14.4 \%$, at the first admission, and an additional $7.5 \%$ of the patients developed lung

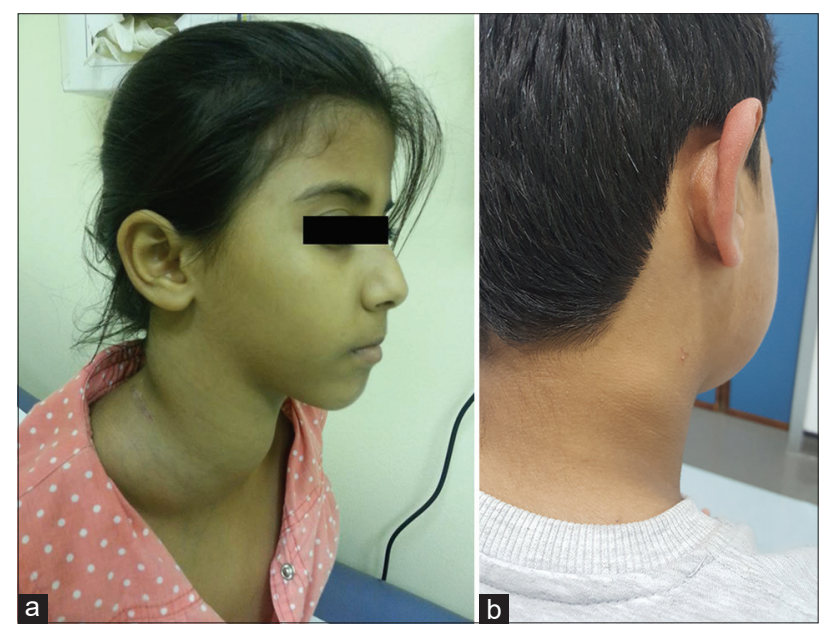

Figure 2: (a) Nine years old girl with huge and bulky right-side confluent anterior and posterior cervical triangle single lymph node mass extending from behind the right ear into the right supra - clavicular and retro - clavicular areas. Histopathology confirmed Classical Hodgkin lymphoma. (b): An 8 years old boy with enlarged firm right upper posterior cervical small lymph node mass $(4 \mathrm{~cm} \times 3 \mathrm{~cm})$. Histopathology confirmed nodular lymphocyte-predominant Hodgkin lymphoma lesions during the course of the disease. As a rule, lung involvement was only observed in advanced stage disease. There is no difference in response to treatment in any specific type of lung lesions. Of all methods, CMT showed the best treatment results. ${ }^{[2]}$

\section{Bone involvement}

Osseous involvement in HL is uncommon and is generally associated with poor prognosis. The most common location is the vertebrae, primarily in the thoraco-lumbar region, followed by the pelvis, ribs, femur, sternum, clavicle, and skull in decreasing order. Ha-ou-nou, et al. reported a case of primary osseous HL in a 35-year-old male presenting with osteolytic lesions in the iliac and sacral bones with no other symptoms. Histopathology confirmed HL. All other investigations, including CT scan of the chest, abdomen, and pelvis and BM biopsy showed no evidence of disease. ${ }^{[22]}$

Primary osseous HL in children is exceedingly rare. However, bone metastasis at presentation and in relapse is not uncommon. Singh and Bakhshi, published a report of three children, out of 66 total pediatric HL patients (4.5\%) with bone involvement over a $3 \frac{1}{2} 2$-year period. At the time of relapse, two patients presented with osseous lesions and one had evidence of nonosseous disease in addition to the bony lesion. Boys and girls were nearly equally affected, local bone pain is the most common symptom, and B-symptoms are common. The vertebrae and pelvic bones were most frequently involved. Commonly with an osteolytic bone picture, NS was the predominant histological subtype. Most children received CMT, and the overall response and OS were very good. ${ }^{[23]}$

\section{Bone marrow involvement}

$\mathrm{BM}$ involvement with $\mathrm{HL}$ at initial presentation is uncommon in pediatric patients. BM involvement may rarely occur as an isolated site for extranodal disease. BM infiltration with HL may take diffuse or focal form with variable number of typical and a typical HRS cell, and is often accompanied by significant reversible marrow fibrosis [Figure 3a-c]. Therefore, BM aspiration is often negative, and BM biopsy is needed. BM involvement is very rare in newly diagnosed low-stage HL (stage I and II) and without systemic B symptoms. BM biopsy should be performed in all patient with advanced stage (clinical Stages III and IV) and B symptoms and in patients undergoing assessment for recurrent disease. Historically, evaluation for skeletal metastasis included ${ }^{99}$ technetium nuclear bone scan and radiography. The recent introduction of the more sensitive ${ }^{18}$ fluorodeoxyglucose positron emission tomography (FDG-PET) scan which accurately assesses both cortical bone and BM may ultimately replace all previously used radiological modalities in detecting bone involvement and replace BM biopsy for the detection of BM infiltration. ${ }^{[16]}$ 


\section{Central nervous system involvement}

Primary central nervous system (CNS) HL is very uncommon. CNS involvement usually occurs in adult patients with advanced or relapsing disease. CNS involvement was reported to occur in $0.2 \%-0.5 \%$ of patients with systemic HL. There are only a few reports of HL involving the CNS concurrently with systemic disease in children. Cerebrospinal fluid sampling provides information about the presence or absence of lymphoma cells but may not be able to further specify the malignant population. Therefore, tissue sampling is required to make accurate diagnosis whenever possible. ${ }^{[24]}$ Van Blydenstein et al. from the University of Witwatersrand in South Africa published a case report of a 12-year-old boy with a history of painless left inguinal swelling and acute diplopia. An intensely enhancing lesion in the right midbrain was detected on MRI scan. The patient was diagnosed with stage IV, NLPHL HL. He was successfully treated with CTR including intrathecal methotrexate (MTX) followed by RT to the brain lesion and remained in remission. ${ }^{[25,26]}$

\section{Skin involvement}

Specific cutaneous involvement in HL is rare and mostly affects adult patients. The mechanisms implicated are

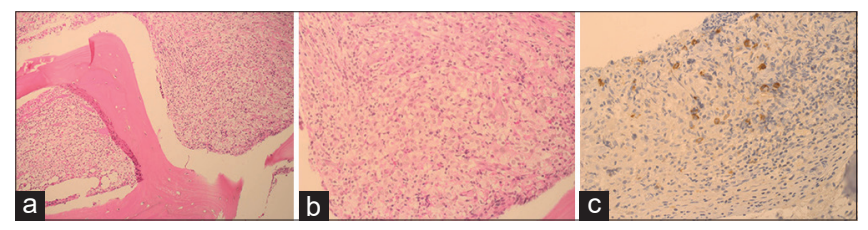

Figure 3: (a) Bone marrow biopsy samples showing cellular marrow with extensive infiltration with monotonous lymphoid hematopoietic cells in a very dense fibrous background $(H$ and $E, \times 20)$. (b) The marrow is infiltrated with sparse large mononuclear cells with large dense nuclei and abundant cytoplasm. Some of these cells has two nuclei giving an impression of Hodgkin and reed-sternberg cells (H and E, ×40). (c) Immunohistochemistry: shows the Hodgkin and reed-sternberg cells stain strongly positive for CD30 immune - stain retrograde lymphatic spread, direct extension from the underlying LN, or hematogenous dissemination. The most common clinical presentation is of single or multiple dermal or subcutaneous nodules. The prognosis of HL with skin infiltration is felt to be extremely poor. However, among the 349 patients reported by Tassies et al., three $(0.85 \%)$ presented with specific cutaneous involvement. In two cases with MC subtype, skin lesions displayed histologic features similar to those found in the LNs. Treatment with combination CTR resulted in rapid disappearance of the lesions and durable remission. ${ }^{[27]}$

\section{Staging system}

The currently used Ann Arbor staging system for HL was adopted and introduced for clinical use by the Committee on HL Staging Classification in 1971 [Table 1]. This staging system has totally replaced the previously used systems and is mainly based on the observation that HL spread appear to run along contagious LNs. The substage classification (A, $\mathrm{B}$ and $\mathrm{E}$ ) indicated certain preset defined clinical features. Substage A indicated asymptomatic disease. B symptoms include fever $\geq 38^{\circ} \mathrm{C}$ for 3 consecutive days, drenching night sweats, and an unexplained body weight loss over the preceding 6 months $(>10 \%)$. Subset E denotes extranodal tissue involvement arising from any contagious nodal region. Subset $\mathrm{S}$ denotes involvement of the spleen. ${ }^{[28]}$

\section{The Use of Computed Tomography and Magnetic Resonance Image Scans for Staging in Hodgkin Lymphoma}

Plain X-ray images have long been used to determine LN and tissue involvement with HL especially in the lungs and mediastinum [Figure $4 \mathrm{a}$ and b]. CT anatomical imaging scan has now almost totally replaced plain X-rays in defining tissue organ involvement. CT scan provides highly accurate information about soft tissue and bone involvement

Table 1: Ann arbor staging system

\begin{tabular}{|c|c|}
\hline Stage & Description \\
\hline $\mathrm{I}$ & $\begin{array}{l}\text { Involvement of single lymph node region or lymphoid structure (e.g. spleen, thymus, Waldeyer's ring), or single extra - } \\
\text { lymphatic site (IE) }\end{array}$ \\
\hline II & $\begin{array}{l}\text { Involvement of two or more lymph node regions on the same site of the diaphragm or localized contiguous involvement of only } \\
\text { extranodal organ/site and lymph node region on the same side of the diaphragm (IIE) }\end{array}$ \\
\hline III & $\begin{array}{l}\text { Involvement of lymph node regions on both sides of the diaphragm (III) which may be accompanied by involvement of the } \\
\text { spleen (IIIs) or by localized contiguous involvement of only one extranodal organ site (IIIE) or both (IIISE) }\end{array}$ \\
\hline III1 & With or without involvement of the splenic hilar, celiac or mesenteric lymph nodes \\
\hline III2 & With involvement of paraaortic, iliac or mesenteric nodes \\
\hline \multirow[t]{2}{*}{ IV } & $\begin{array}{l}\text { Diffuse or disseminated involvement of one or more extranodal organs or tissue, with or without associated lymph node } \\
\text { involvement }\end{array}$ \\
\hline & Designations applicable to any stage \\
\hline A & No symptoms \\
\hline B & $\begin{array}{l}\text { Fever (temperature } \geq 38^{\circ} \mathrm{C} \text { ) for } 3 \text { days, drenching night sweats, unexplained weight loss }>10 \% \text { of the body weight within the } \\
\text { preceding } 6 \text { months }\end{array}$ \\
\hline $\mathrm{E}$ & Involvement of single extranodal site that is contagious or proximal to the known involved nodal site \\
\hline $\mathrm{S}$ & Splenic involvement of any pattern (nodular, patchy, or diffuse) \\
\hline
\end{tabular}


with lymphoma in nearly all body sites [Figure 5a-d]. The use of functional nuclear scans such as gallium scan has significantly helped in defining tissue involvement with HL [Figure 6]. More recently, FDG-PET-CT scan was used to detect tissue involvement with greater accuracy. FDG-PET-MRI scans have been recently introduced in the primary investigations for HL in adult patients. PET-MRI scans were shown to be a reliable alternative to PET-CT scans. PET-MRI has comparable accuracy to PET-CT scans in detecting the disease sites but added the benefit of RT dose reduction. Nowadays, the use of FDG-PET-CT scans for disease staging and response assessment has become the standard of care for providing optimal therapy for pediatric patients with $\mathrm{HL}^{[29,30]}$ [Figure 7].

\section{The Use of ${ }^{18}$ Fluorodeoxyglucose Positron Emission Tomography and Gallium Scans}

The use of functional nuclear scans such as the gallium and FDG-PET scans has increased the accuracy of detecting tissue involvement with HL. FDG-PET scan seems to have slightly higher sensitivity to gallium scan. FDG-PET scan is considered nowadays as the most valuable imaging
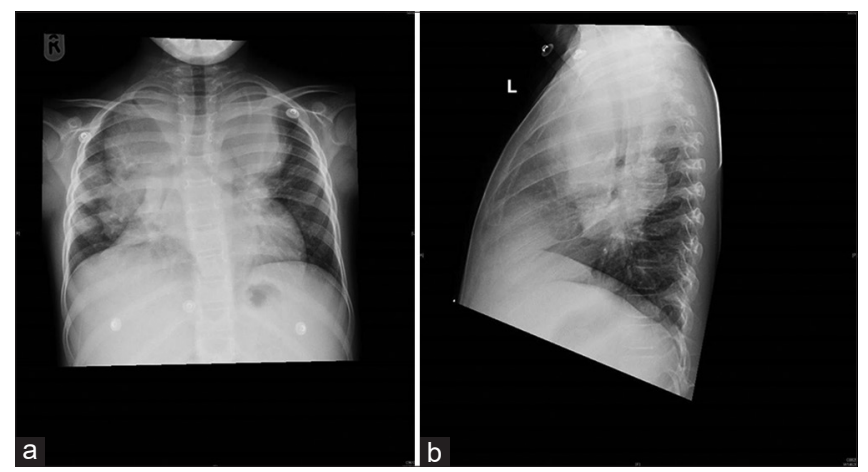

Figure 4: Plain CXR films, anterio - posterior (a) and lateral (b) views, from a patient with classical Hodgkin lymphoma showing huge bilateral anterior mediastinal and hilar lymphadenopathy

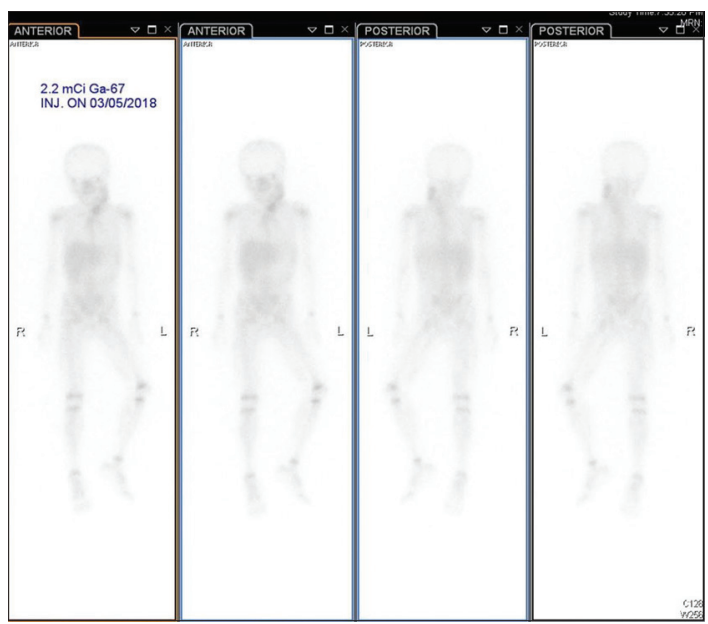

Figure 6: Gallium - 67 nuclear scan from a patient with classical Hodgkin lymphoma, showing a large area of increased gallium in the left cervical lymph node group extending down to the left supraclavicular region, the upper anterior mediastinum and the left lung hilum tool in HL [Figure 6]. Since its introduction in the early 1990 s, it has subsequently become the gold standard in the initial staging, interim, and end of treatment remission assessment. ${ }^{[29]}$ Response assessment by FDG-PET-CT scan may be particularly important as it allows early modification to maximize treatment efficacy. Data demonstrated significant value of gallium scintigraphy scan in accurate staging and response assessment in pediatric

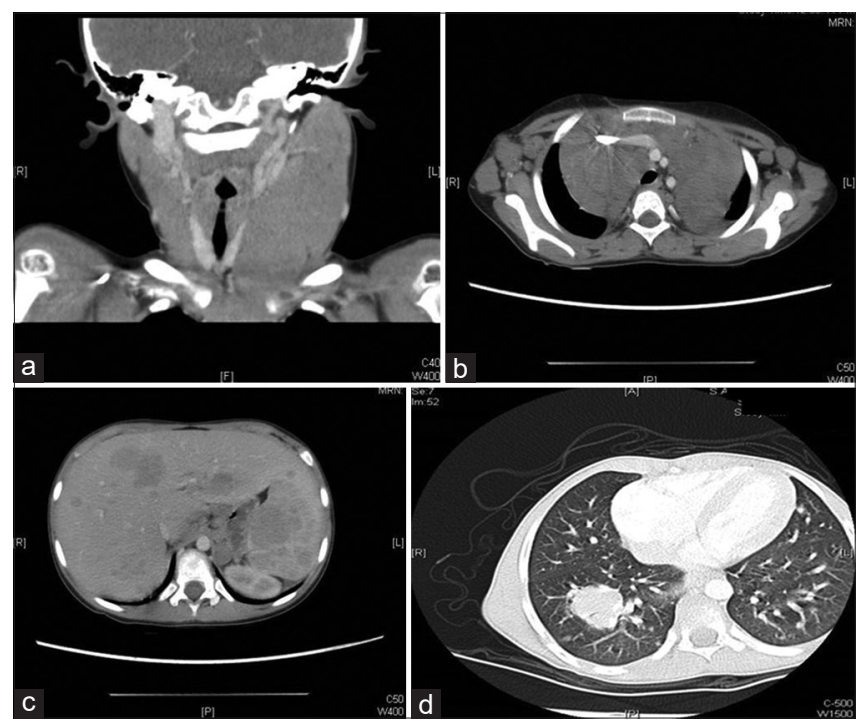

Figure 5: (a) Noncontrast computed tomography scan films of the neck from a patient with classical Hodgkin lymphoma, showing hugely enlarged cervical lymph nodes more prominent in the left side. (b) Computed tomography scan of the chest from a patient with classical Hodgkin lymphoma showing bilateral enlarged anterior and posterior mediastinal lymph nodes. (c) Computed tomography scan of the abdomen. The liver and spleen are enlarged and infiltrated with multiple variable size hypodense focal lesion in keeping with Hodgkin lymphoma infiltrates. In addition multiple enlarged lymph nodesare seen in the mesentery and the porta - hepatis. (d) Computed tomography scan of the chest. The lungs windows show multiple scattered variable size bilateral pulmonary nodules, the largest is seen in the right lower lobe measuring $2.9 \mathrm{~cm} \times 2.5 \mathrm{~cm}$ indicating lung metastasis

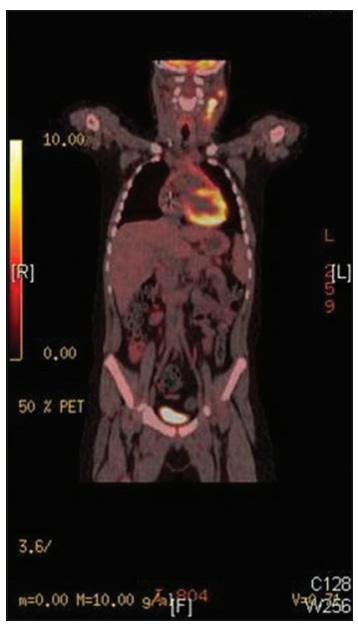

Figure 7: 18-fluorodeoxyglucose positron emission tomography/computed tomography scan from a patient with classical Hodgkin lymphoma, showing increased 18-fluorodeoxyglucose uptake in the left side of the neck indicating disease activity. The mild increase 18-fluorodeoxyglucose uptake in the thymus indicates physiological activity 
patients with HL. In addition, evaluation of gallium positivity is very useful prognostic parameter. Positive gallium or FDG-PET scan at the end of first-line therapy retains of great significance in terms of EFS and OS. ${ }^{[31-33]}$ The International Harmonization Project defined complete response (CR) after treatment for HL by absence of FDG-PET avidity at the end of CTR, regardless of the size of residual soft tissue masses. Residual avidity after initial treatment is known to predict inferior outcomes. ${ }^{[30,34]}$

\section{Change of Chemotherapy Regimens over Time}

The goal of treatment of pediatric and adolescent patients with HL is to achieve cure without significant long-term morbidity. Earlier management approaches used invasive staging systems and high-dose RT and CTR for treatment without particularly considering long-term side-effects. Some children suffered devastating complications such as severe growth and developmental issues, muskeloskletal hypoplasia, cardiovascular and pulmonary dysfunction, and second malignant neoplasms (SMN). Subsequent recognition of the life-threatening and life-altering complications led to the development of CMT regimens with restriction of RT doses and fields and avoiding excessive use of alkylating agents. Progress in imaging techniques eventually obviated the need for use of surgical staging procedures. Over time, researches made further modification that decreased the number of CTR cycles and eliminated specific agents that predisposed to treatment associated toxicities. ${ }^{[35-37]}$

The MOPP (mechlorethamine, vincristine [VCR], procarbazine $[\mathrm{PZN}]$, prednisone [PSN]) combination was first introduced in the early 1960s and was the first successful CTR regimen in both adult and pediatric HL. The MOPP regimen has the longest period of follow-up to date and is best studied as to its benefits and complications. Acute toxicities such as nausea, vomiting, mucositis, hair loss, and myelosuppression, justified the modification of doses of nitrogen mustard, an agent whose dose intensity is crucial in achieving cure. Substitution of mechlorethamine with chlorambucil (ChL) and vinblastine (VBL) in the ChlVPP (ChL, VBL, PZN, PSN) regimen virtually relieved all acute toxicities, except myelosuppression. However, the long-term toxicity of sterility (especially in males) and myelodysplasia were most likely related to alkylating agent and would had not been influenced by the use of various variant of MOPP such as COPP (ChL, VCR, PZN, PSN), MVPP (mechlorethamine, VBL, PZN, PSN), and ChlVPP. ${ }^{[38,39]}$

Doxorubicin (DOX)-containing regimens, such as ABVD (DOX, bleomycin [BCN], VBL, dacarbazine [DZN]), and ABDLP (DOX, BCN, DZN, lomustine, PSN) have significant antitumor effect but have been initially used as second-line therapy. ABVD has been incorporated into the MOPP/ABVD schemes and into hybrid regimens that attempt to offer all the antilymphoma active agents together, such as the MOPP/ABV. The initial experience has been encouraging with high and sustainable remission rates. MOPP/ABVD $\times 6$, have been compared with MOPP $\times 6$ with the alternating regimen showing significant superiority. The Cancer and Leukemia Group has compared the use of MOPP with MOPP/ABVD and a third arm ABVD alone for the initial treatment of HL. The CR and failure rates for MOPP/ABVD and ABVD alone were superior to the MOPP. The significant modifications made in the doses of the MOPP components may explain the differences, since only $20 \%$ of patients were receiving the full doses of nitrogen mustard by the sixth dose. The ABVD, however, has a different toxicity profile, where myelodysplasia and sterility are nearly not seen, but small number of patients may develop pulmonary fibrosis due to the use of BCN and RT. The question that always comes to mind is whether ABVD can be improved? Etoposide has very demonstrated very good single agent activity in HL and that has prompted its inclusion in some second-line regimens, such as EVAD (etoposide [VP16], VCR or VBL, DOX). The second-line CR rates in the St. Bartholomew's hospital series, London, England (where etoposide was used), was 11 of 19 patients $(58 \%))^{[38]}$

The Van den Berg study reported a group of 21 children ( 7 females and 14 males) aged 5-18 years (median 14 years), diagnosed with HL between 1988 and 1993. Their clinical stages were stage 1, 7 patients; stage II, 8 patients; stage III, 5 patients; and stage IV, 1 patient. Their pathology revealed $2 \mathrm{LP}, 17 \mathrm{NS}$, and $1 \mathrm{MC}$. One patient, had only cytology done, thus histopathologic subclassification was not possible. All children were treated with alternating MOPP and ABVD cycles $(3 \times$ MOPP, $3 \times$ ABVD). Two children have relapsed giving a disease-free survival (DFS) of $90 \%$. Analysis of side effects revealed no cardiac or pulmonary toxicity. Out of ten patients tested, one patient had mild hypogonadism and no patient developed SMN. Although the number was small, the study concluded that treatment with MOPP/ABVD for 6 cycles without RT may be adequate. ${ }^{[39]}$

The role and timing of RT in the treatment program of pediatric HL seems to have been well established. Current treatment options for pediatric HL include combining IFRT with CTR regimens such as the ABVD, ABVE-PC (DOX, BCN, VCR, VP16, PSN and cyclophosphamide [CPA]) and COPP as CMT, which provides optimum long-term survival for $90 \%-95 \%$ of patients but still associated with risks of late effects of RT. Use of ABVD as a single modality therapy without RT was associated with higher risk of disease recurrence $(5 \%-7 \%$ greater than that observed with CMT alone), but avoids the RT long-term side effects. ${ }^{[40]}$

\section{The Change from Extended Field to Involved-Field Radiotherapy}

In the mid- $20^{\text {th }}$ century, the standard management for HL cases was initial staging using laparotomy and splenectomy 
followed by high-dose extended field (MANTLE and Inverted Y) RT (36-44 Gy) with or without the addition of CTR. This approach resulted in dramatic rise in the OS and EFS over the subsequent few years. However, associated with this improvement came the substantial adverse sequelae observed in children who survived the disease. ${ }^{[41]}$ This observation led to reduction of RT dose and field for the growing children and introduction of more effective less toxic CTR combinations. Over the subsequent years, a more refined CTR- RT combinations were introduced, which maintained the survival rates and reduced the frequency of the short- and long-term toxicities. ${ }^{[42]}$ RT, however, remains the standard of care in most institutions, but, nowadays, it is only given to specific risk groups of patients such as those who has bulky disease at presentation and fails to show good response to induction CTR. With the ability to precisely define the sites of tissue involvement using modern radiological tests, involved and limited field RT were substituted for the earlier extended RT fields. The current treatment option of combining IFRT with CTR enabled reduction of the RT dose, field, and volume without reducing the cure rates..$^{[40,41]}$

\section{Prognostic Criteria in Children}

Advances in the treatment of pediatric HL have led to reduction or even loss of significance of many of the previously known prognostic factors. Yet, some prognostic factors remain useful in guiding therapy by defining risk stratification and predicting the final outcomes. Prognostic factors in HL can be divided into those which are patient related (e.g., age, gender) and those related to the tumor (e.g., pathological subtype, disease extent, bulk). The degree at which prognostic factors (e.g., disease stage, bulk, and presence of B symptoms) are interrelated with therapy will determine their applicability. Precise definition of the risk factor (e.g., bulk disease, sites of involvement, age cutoffs, relevant range of laboratory values such as erythrocytic sedimentation rate [ESR], serum lactate dehydrogenase and serum ferritin) is of great importance to made proper interpretation of the results..$^{[43]}$

The Children Oncology Group (COG) developed a predictive model for EFS in pediatric/adolescent HL using clinical data known at diagnosis from 1103 intermediate-risk HL patients treated according to the COG AHOD0031 protocol using the combination of ABVE-PC CTR and IFRT. Independent predictors of EFS were identified and used to develop and validate the new COG Childhood Hodgkin International Prognostic Score (CHIPS). A study cohort including approximately half of the overall cohort was randomly selected, with the remainder forming the validation cohort. Stage IV disease, large mediastinal mass, albumin $(<3.5 \mathrm{mg} / \mathrm{dl})$, and fever were independent predictors of EFS. Each factor was assigned 1 point in the CHIPS. For patients with CHIPS $=0$, the 4 -year EFS was $93.1 \%, 88.5 \%$ for patients with CHIPS $=1,77.6 \%$ for patients with CHIPS $=2$, and $69.2 \%$ for patients with CHIPS $=3$. The study concluded that CHIPS was highly predictive of EFS. The study identified a subset of (patients with CHIPS $=2$ or 3 ) that comprises $27 \%$ of intermediate-risk group who have a 4-year EFS of $<80 \%$. Those patients may benefit from early therapy augmentation. CHIPS has also identified higher risk group of patients who were not identified by early FDG-PET or CT scan response. CHIPS proved to be a robust and inexpensive tool in predicting risk in patients with intermediate-risk HL. This that may improve ability to tailor therapy to risk factors known at diagnosis..$^{[44,45]}$

\section{Risk-Based Therapy}

\section{Low-risk Hodgkin lymphoma therapy}

Patient with early-stage HL (stage I and stage II without bulky disease or B symptoms) has low relapse probability with EFS $>85 \%$ and OS $>95 \%$. Therefore, recent treatment strategies have focused on maintaining high EFS and OS while minimizing the use of therapy to reduce early and late side effects. Strategies used to treat children with HL vary greatly between the pediatric cooperative groups and there is no one standard treatment. ${ }^{[44,45]}$ To minimize the late effects of treatment, trials were conducted where RT was omitted in patients in CR after 2-3 cycles of CTR. Furthermore, the standard dose of RT was reduced to 20 Gy in patients in less than CR. The German Society of Pediatric Oncology published the results of the HL trial 95 which included 925 pediatric patients with classical HL treated between 1995 and 2001 in seven European countries. Patients in treatment group (TG) 1 (TG1; early stages) received two cycles of VCR, PSN, PZN, and DOX (OPPA) or VCR, PSN, VP16, and DOX (OEPA) CTR. Additional two or four cycles of CPA, VCR, PSN, and PZN (COPP) were added in TG2 (intermediate stages) or TG3 (advanced stages), respectively. Patients in CR (assessed by CT or MRI scans) did not receive RT. Those with tumor volume reduction $>75 \%$ received reduced dose IFRT $(20 \mathrm{~Gy}$ ) and an additional 10-15 Gy boost only for larger residuals. The study showed very good results with the OS, progression-free survival (PFS), and EFS at 10 years were ( \pm standard error) $96.3 \% \pm 0.6 \%, 88.2 \% \pm 1.1 \%$, and $85.4 \% \pm 1.3 \%$, respectively. PFS for TG1 patients with or without receiving RT was $97.0 \% \pm 2.1 \%$ versus $92.2 \% \pm 1.7 \%(P=0.214)$. Results were not satisfactory for nonirradiated patients in TG2 $(68.5 \% \pm 7.4 \%$ vs. $91.4 \% \pm 1.9 \% ; P<0.0001)$, with similar but not significant results in TG3 $(82.6 \% \pm 5.4 \%$ vs. $88.7 \% \pm 2.0 \%$, $P=0.259)$. Reduction of the standard RT dose from 25 to 20 Gy did not increase relapse rate. The study concluded that RT can be safely omitted in early stage HL in CR following CTR. RT with (20-35 Gy) proved to be sufficient in patients with $<\mathrm{CR}$ following CTR. ${ }^{[45]}$ Radford et al. reported the outcome of the randomized phase III trial to determine the role of FDG-PET imaging in clinical stages 
IA/IIA HL (The RAPID study). The study was conducted in 94 centers across the United Kingdom. Patients aged 16-65 years with newly diagnosed stage IA or stage IIA $\mathrm{HL}$ received three cycles of $\mathrm{ABVD}$ and then underwent FDG-PET scanning. Patients with negative FDG-PET were randomly assigned to receive IFRT or no further treatment; patients with positive FDG-PET received a fourth cycle of ABVD and RT. A total of 602 patients $(53.3 \%$ male; median age, 34 years) were enrolled, and 571 patients (95\%) underwent FDG-PET scanning. The FDG-PET findings were negative in 426 patients $(74.6 \%)$, $420(98.6 \%)$ of whom were randomly assigned to a study group (209 to the RT group and 211 to no further therapy). At a median follow-up of 60 months, eight patients (3.8\%) developed disease progression in the RT group and eight patients had died (three with disease progression). Twenty patients $(9.5 \%)$ developed disease progression in the group who received no further therapy, and 4 patients $(25 \%)$ had died. The 3-year PFS rate was $94.6 \%$ (95\% confidence interval [CI], 91.5-97.7) in the RT group and $90.8 \%(95 \%$ CI, 86.9-94.8) in the group that received no further therapy. The results of this study showed no inferior outcomes in patients who received no further treatment after CTR with regard to PFS. Nevertheless, in this study, patients with early stage HL and negative FDG-PET scan after three cycles of ABVD had very good prognosis either with or without RT. ${ }^{[46-48]}$

The COG has recently published the results of the AHOD0431 study for treatment of low stage, low risk HL. The study enrolled 278 patients $<21$ years old and evaluated a response-based treatment plan where minimal CTR was given. Low-dose IFRT was given only to patients who did not achieve CR. A combination of CTR and low-dose IFRT salvage regimen was given to those who had low-risk recurrence. At 4 years, $49.0 \%$ had received minimal CRT and no RT, $88.8 \%$ were in remission without receiving autologous blood and marrow transplantation (ABMT) or $>21$ Gy of IFXRT. The OS rate was $99.6 \%$. The study was closed early when the recipients of RT exceeded the predefined monitoring boundary. This limited CTR response-based approach was successful in patients who had a negative FDG-PET1 result, MC histology, or a low ESR. Donaldson SS reported the results of treatment of 110 pediatric patients with low-risk HL using four cycles of VBL, DOX, MTX, and PSN (VAMP) and 15 Gy IFRT for those who achieved CR or 25.5 Gy for those who achieve partial response after two cycles of VAMP. After a median follow-up of 9.6 years (range, 1.7-15.0 years), 5- and 10 -year OS were $99.1 \%$ and $96.1 \%$, respectively, and 5- and 10-year EFS were $92.7 \%$ and $89.4 \%$, respectively. Factors contributing to 10 -year EFS were early CR $(P=0.02)$, absence of B symptoms $(P=0.01)$, LP histologic subtype $(P=0.04)$, and less than three initial sites of disease $(P=0.02)$. Organ toxicity was limited to hypothyroidism in $42 \%$ of irradiated patients, and one case of cardiomyopathy. Seventeen healthy babies have been born to 106 of the survivors. Two patients developed SMN: one thyroid cancer within the RT field and one Ewing's sarcoma outside the RT field. The study proved that pediatric patients with low-risk HL can be cured using CTR regimens without alkylating agents, BCN, VP16, or high-dose RT. Acute and long-term toxicities were minimal. ${ }^{[48,49]}$

\section{Intermediate-risk Hodgkin lymphoma therapy}

The COG AHOD0031, a randomized Phase III study, was designed to evaluate the role of early CTR response in tailoring subsequent therapy in patients with pediatric intermediate-risk HL. Patients received two cycles of ABVE-PC combination followed by CT scan evaluation. Rapid early responders (RERs) received two additional ABVE-PC cycles of CTR, followed by response evaluation. RERs with $\mathrm{CR}$ were randomly assigned to receive IFRT or no additional therapy; RERs with less than CR were non-randomly assigned to IFRT. Slow early responders (SERs) were randomly assigned to receive additional two cycles of ABVE-PC with or without additional two cycles of dexamethasone, VP16, cisplatin (CIS), and cytarabine (DECA) CTR. All SERs received IFRT. The study showed very good results where the 4-year EFS was $85.0 \%: 86.9 \%$ for RERs and $77.4 \%$ for SER's $(P<0.001)$. The 4 -year OS was $97.8 \%$ for RERs and $95.3 \%$ for SERs $(P<0.001)$. The 4 -year EFS was $87.9 \%$ versus $84.3 \%(P<0.11)$ for RERs with $\mathrm{CR}$ who were randomly assigned to IFRT versus no IFRT, and $86.7 \%$ versus $87.3 \%(P<0.87)$ for RER's with FDG-PET scan - negative patients at second response assessment. The Four-year EFS was $79.3 \%$ versus $75.2 \%(P<0.11)$ for SER's who received DECA versus those who did not and $70.7 \%$ versus $54.6 \%(P<0.05)$ for SER patients with FDG-PET-positive results at second response assessment. This study demonstrated that early response assessment supported therapeutic modification. The study has also demonstrated that although response-based therapy has helped establishing optimal treatment for selected RER patients, it has not helped in improving outcome for SER patients or clearly defined the IFRT volumes or doses. ${ }^{[50]}$

\section{High-risk Hodgkin lymphoma therapy}

Upfront dose-intensified treatment strategies for HL demonstrated improved cure rates compared to earlier studies despite the increased risk of acute and long-term toxicities. Multiple trials have clearly demonstrated that ABVD was as good as the MOPP or even better and with less toxicity profile. The ABVD (or one of its variants) has become the most commonly used regimen for the treatment of adult and pediatric patients with advanced-stage (stage III and IV) HL in North America. Although $60 \%-70 \%$ of patients with advanced stages HL were cured with ABVD, many patients still relapse or progress on therapy. ${ }^{[51]}$ More recently, the German Hodgkin Lymphoma Study Group 
has developed a front-line intensified regimen for adult patients with HL consisting of BCN, VP16, DOX, CPA, VCR, PZN, and PSN (BEACOPP), which includes higher-than-standard doses of VP16, DOX, and CPA. This regimen, as compared with COPP-ABVD, has led to better tumor control and to an increase in OS at 10 years by $11 \%$. The choice of a first-line treatment between the different available CTR regimens requires balancing cure with the occurrence of early and late complications. To properly consider this balance, the treatment decision should take into consideration the outcome after using a second-line therapy (should relapse or progression occur), especially when effective salvage regimens are available. ${ }^{[52]}$

The COG assessed the feasibility of a dose-intensive regimen, BEACOPP in children with high-risk HL (Stage IIB, IIIB with bulk disease, Stage III and IV). Response was assessed after 4 cycles of BEACOPP. RERs received sex guided consolidation therapy (to reduce sex-specific long-term toxicities). Females received four cycles of COPP/ABV without RT. Males received two cycles of ABVD followed by IFRT. SERs received four cycles of BEACOPP followed by IFRT. Ninety-nine patients were enrolled. RER was achieved by $74 \%$ of patients. The OS was 97\%. And the Five-year EFS was $94 \%$, with median follow-up of 6.3 years. No disease progressions was observed during the study. Secondary leukemia occurred in two patients. As per the study results, in pediatric patients with high-risk HL, early intensification followed by less intense response-based therapy for RER was very effective in achieving high EFS. ${ }^{[53]}$

The BEACOPP regimen has been advocated as the new standard therapy for advanced stage HL, in place of ABVD. The Italian Cooperative Group has published a study comparing ABVD with BEACOPP for advanced stage HL. A total of 331 patients with previously untreated HL (Stage IIB, IIIA and $\mathrm{B}$, or IV) where randomized to receive either BEACOPP or ABVD, followed by IFRT (when indicated). Patients with residual or progressive disease after the initial therapy were to receive high-dose salvage RT. For a median follow-up of 61 months, the 7-year rate of freedom from first progression was $85 \%$ among patients received initial treatment with BEACOPP and 73\% among patients who received ABVD $(P=0.004)$. The 7-year EFS was $78 \%$ and $71 \%$, respectively $(P=0.15)$. A total of 65 patients (20 in the BEACOPP group, and 45 in the ABVD group) received high-dose salvage RT. Three of the twenty patients in the BEACOPP group and 15 of the 45 in the ABVD group who had had progressive disease or relapse were alive and in remission by the cutoff date. After completion of the whole treatment plan, including salvage therapy, the 7-year rate of freedom from a second progression was $88 \%$ in the BEACOPP group and $82 \%$ in the ABVD group $(P=0.12)$, and the 7-year rate of OS was $89 \%$ and $84 \%$, respectively $(P=0.39)$. Severe therapy-related side effects occurred more frequently in the BEACOPP group than in the ABVD group. The study concluded that, treatment with BEACOPP, as compared with ABVD, resulted in better initial tumor control, but the long-term clinical outcome did not vary between the two regimens. ${ }^{[52]}$

The Pediatric Oncology Group (POG) introduced a treatment regimen for both low-and high-risk HL with ABVE or dose - intensified ABVE with the addition of PSN and CPA (ABVE-PC), followed by low-dose IFRT in a response-based approach. The number of CTR cycles was determined by rapidity of the initial response. Based on the experience of POG 8725 protocol, the POG 9426 study was conducted to test the efficacy of the ABVE backbone for pediatric patients with advanced stage HL (Stage IIIB and Stage VI). The use of the less myelotoxic VCR instead of VBL, commonly used in ABVD allows for escalation DOX and VP-16. The single-agent efficacy of VCR and VBL however are virtually identical. Data from POG 8725 study showed that patients with advanced stage HL who achieve CR after 3 cycles of CTR have $>91 \%$-year EFS. In the POG 9426 study, patients in CR or "probable CR" proceeded directly to IFRT (21 Gy). Others received two additional cycles of ABVE-PC prior to RT. All patients received low dose IFRT (Although POG 8725 has not shown a benefit for RT, but a significantly different CTR was used). The 216 eligible patients were 22 years of age or younger. ABVE-PC was administered every 21 days. RER's to 3 cycles of ABVE-PC received 21 Gy IFRT. RER was documented in $63 \%$ of patients. SER's received 2 additional ABVE-PC cycles before receiving IFRT (21 Gy). The 5-year EFS was $84 \% ; 86 \%$ for the RER and $83 \%$ for the SER $(P=0.85)$. Only $1 \%$ of patients had progressive disease. The 5-year OS was 95\%. ABVE-PC was very effective and provided impressive EFS and OS (with short duration) for pediatric patients with advanced stage HL. ${ }^{[54]}$

\section{Nodular Lymphocyte-Predominant Hodgkin Lymphoma}

NLPHL is a rare variant of HL in children, forming nearly $5 \%$ of all cases. As specific immunohistochemical staining has become available, NLPHL was able to be separated from classical HL more accurately. NLPHL is characterized by the presence of LP cells, which are CD20+ but CD15 and CD30 negative. LP cells are seen scattered amongst group of small B lymphocytes arranged in a nodular pattern. NLPHL often present with limited low stage disease and small non bulky cervical LN mass [Figure 2b]. Extranodal disease and B symptoms are uncommon. However, occasional patients may present with bulky and widespread advanced stage disease. Few reports are available about pediatric NLPHL treated with CTR only. ${ }^{[55,56]}$ Upfront treatment of NLPHL patients consisted of four cycles of CTR such as epirubicin, BCN, VBL and DZN (EBVD) without RT, compared to classic HL patients who receive 4-6 cycles of CTR e.g., EBVD or EBVD/COPP +/-RT. This 
strategy had good success in patients with limited NLPHL but patients with advanced stage tend to relapse therefore need to receive similar CTR and possibly RT to classic HL patients. ${ }^{[57]}$ Limited (stage I and II) NLPHL patients were treated with RT alone. This strategy was more successful in patients with supradaphratic disease as they experienced very low relapse rate but significant long-term side effects. Also, in patients with low stage (stage I) disease, radical surgical resection of the involved LN group was performed, but after few months of observation more than half of the patients developed relapse. ${ }^{[56,58]}$

\section{Relapsed Hodgkin Lymphoma}

Despite improvement in treatment results of all stages of HL, there is still around $15 \%$ of patients who are not cured or relapse after initial response to CTR and/or RT. The outcome of patients with HL who progress or relapse following CTR is poor and depending on certain patient and disease factors the OS ranges between $10 \%$ and $50 \%{ }^{[57]}$ The COG has published the results of the AHOD0031 study that was designed to detect the most common pattern of relapse and whether response-based therapy improves outcomes. From September 2002 to July 2010, 1712 patients $<22$ years with intermediate-risk HL were enrolled and treated with ABVE-PC \pm IFRT. Relapses were characterized without respect to site (initial, new, or both; and initial bulk or initial non-bulk) and IFRT field. Patients were subgrouped by the initial treatment assignment (SER; RER/no CR; RER/CR/IFRT; and RER/CR/no IFRT). At 4-year median follow-up, $244 / 1712$ patients (14.2\%) relapsed, 198 of whom were fully evaluable. The median time to relapse was 12.8 months. Of the 198 evaluable patients, 30\% were RER/no CR, 26\% were SER, 26\% were RER/CR/no IFRT, 16\% were RER/CR/IFRT, and 2\% uncategorized. Relapse involved $74 \%$ and $75 \%$ of initially bulky and nonbulky sites, respectively. ${ }^{[50,59]}$

Unfortunately, conventional standard-dose salvage CTR for relapsed disease has disappointing results in terms of OS. Salvage CTR regimens such as DECA or EPIC (VP16, CIS, ifosfamide, and cytarabine) were used with variable results. The addition of RT consolidation especially to previously nonirradiated sites may improve the outcomes. While there is no standard of care in terms of salvage CTR, consolidation with ABMT has become the standard of care despite lacking evidence of its superiority to conventional CTR \pm RT. However, nearly $45 \%$ of patients relapse after $\mathrm{ABMT} .^{[60]}$ In general, patients with late relapse (>12 months after completion of therapy) may be cured with conventional therapy. Patients with progressive disease and those with early relapse (3-12 months) are considered candidates for ABMT. Predictive factors such as refractory disease, relapse within 12 months of completing therapy, Ann Arbor staging at relapse, and relapse in a previously irradiated field classically are used to identify patients with poor outcomes. According to patient selection criteria, OS and DFS rates after ABMT are 43\%-95\% and $31 \%-70 \%$, respectively. ${ }^{[60]}$

Scarce data are available on the use allogeneic BMT for treatment of children with relapsed HL. Broader use of allogenic BMT has been hampered by the associated high nonrelapse mortality, overshadowing the advantage of the resultant graft-vs-lymphoma effect. Data suggest that young patients with recurring disease following ABMT, some patients with multiple relapses and patients with refractory HL, may benefit from allogeneic BMT, but the risk of further relapses remain significant. ${ }^{[59,61]}$

With time, investigators are learning more about the biologic mechanisms involved in the pathogenesis of HL and more biologically based therapies are being investigated and introduced to treat patients at diagnosis and at relapse. Both specific small molecules targeted therapies and immunotherapy are being studied as possible treatment options. Although the majority of studies on novel agents took place in adult patient settings, pediatric and adult HL virtually have similar pathology and biology, therefore, study results may potentially be applied to pediatric patients. ${ }^{[31]}$ The COG conducted a Phase II study to assess the efficacy and toxicity of gemcitabine and vinorelbine $(\mathrm{GV})$ in thirty pediatric patients with relapsed/ refractory HL. Vinorelbine $25 \mathrm{mg} / \mathrm{m}^{2} /$ dose and gemcitabine $1000 \mathrm{mg} / \mathrm{m}^{2} /$ dose IV were given every 3 weeks. Response was evaluated after every two cycles. All patients were treated in the past with at least two prior CTR regimens, and 17 patients received prior ABMT. Hematologic toxicity was predominant, but there were no toxic deaths. Measurable responses were seen in $19(76 \%)$ of 25 assessable patients (95\% CI, 55\% to 91\%). Six patients achieved CR, 11 very good partial Response, and two partial responses (PR). Therefore, GV was considered as effective regimen for children with relapsed or refractory HL and with low toxicity profile. ${ }^{[62]}$ Brentuximab vedotin, an antibody drug conjugate that targets CD30 receptors, became the first drug to be approved by regulatory agencies for the treatment of HL in adult patients. ${ }^{[63]}$ The drug is used exclusively in CD30 + classical HL patient with relapsed/refractory disease, mainly as a third-line salvage therapy following ABMT. ${ }^{[64]}$ The drug was also successfully used as second-line therapy before ABMT was performed. ${ }^{[63]}$ The COG conducted a single-arm, phase 1-2 clinical trial, in pediatric and young adults with relapsed/ progressive HL. All patients had primary refractory disease or relapse $<1$ year from completion of initial treatment. Each 21-day cycle consisted of $1000 \mathrm{mg} / \mathrm{m}^{2}$ intravenous gemcitabine on days 1 and 8 and intravenous brentuximab vedotin on day 1 at $1.4-1.8 \mathrm{mg} / \mathrm{kg} /$ dose. The primary objectives were to establish the recommended phase 2 dose of brentuximab vedotin in this combination and the safety profile. Forty-six patients were enrolled, including one who was found to be ineligible, in the two phases of the study. FDG-PET-CT scan was used for response evaluation. 
Twenty-four (57\%) of 42 evaluable patients (95\% CI 41-72) had a CR within the first four cycles of treatment. Four patients had either PR or stable disease. The total number with CR was 28 [67\%] of 42 [95\% CI 51-80]). There were no treatment-related deaths. Brentuximab vedotin with gemcitabine was found to be an effective and safe treatment for patients with relapse/refractory HL. ${ }^{[34,65,66]}$

\section{Current Treatment Strategies and Prognosis}

Nowadays and using the pretreatment risk stratification and timely response assessment with the modern radiology tests and treatment modification accordingly, HL has become one of the most curable pediatric and adult cancers. More than $90 \%$ of patients with limited-stage and nearly $80 \%$ of patients with advanced-stage HL achieve cute and long-term DFS using CTR alone or combination of CTR and RT. Global collaboration in clinical trials within cooperative pediatric HL study groups in Europe, Australasia, and North America has resulted in continued improvement; however, survivors of pediatric HL are still at high risk of developing SMN's and cardiovascular complications. ${ }^{[67]}$

Over the last three decades, all major international pediatric and several adult HL study groups have followed the risk-adopted response-based treatment strategy and toxicity sparing through rationalizing the use of RT. In low-risk patients, multiple studies have been conducted to investigate limiting RT, CTR, or both to prevent long-term side effects without affecting the excellent cure rate achieved. In intermediate- and high-risk HL patients, many studies have examined intensifying upfront and subsequent therapy to improve EFS rates ${ }^{[31]}$ High treatment efficacy was achieved using upfront dose intensified CTR. Refinement of CTR and RT has been implemented, where RT was completely eliminated from the treatment plan of subgroups of patients who showed CR to induction CTR. ${ }^{[50]}$

Because the pediatric staging and response assessment criteria are not uniform, comparing the results of trials among different pediatric and adult study groups remains difficult; thus, initiatives are desperately needed to standardize therapeutic risk stratification and response definitions. ${ }^{[67,68]}$

\section{Follow-up Radiological Tests}

In following up HL patients, in the first 5 years, the main focus is to detect recurrence, while after 5 years, the focus is on detecting the late effects of treatment. Children with HL routinely undergo follow-up CT scans and sometimes gallium and FDG-PET-CT scans performed at different frequencies for up to 5 years after treatment completion. A number of recent studies have demonstrated that routine surveillance imaging, by CT, FDG-PET-CT, as well as other imaging techniques, may be overutilized and contribute to increased cost and RT exposure with no clear benefits. ${ }^{[69]}$ Friedmann et al. reviewed the outcomes of pediatric patients with $\mathrm{HL}$ treated according to $\mathrm{COG}$ protocols between 1990 and 2006 to determine the primary event that led to the detection of relapse. Relapse occurred in 64 of 402 evaluable patients $(15.9 \%)$ at a median of 1.7 years from the time of diagnosis. The majority of relapses $(47 \%)$ were diagnosed at a routine visit, and patient complaint was the initial finding that led to a diagnosis of relapse. An abnormal finding on physical examination was the primary event in another $17 \%$ of relapses, and imaging abnormalities led to the diagnosis of relapse in the remaining $36 \%$ of patients. Laboratory abnormalities were never the primary finding and the method and timing of detection of relapse (whether detected at a routine visit or an extra visit) had no significant impact on survival.

The COG published a study that included $216 \mathrm{HL}$ patients, age $\leq 21$ years old, treated according to the POG 9425 trial. Data for patients who experienced relapse were retrospectively reviewed to determine whether imaging or clinical events prompted suspicion of relapse. Results showed that 25 patients (11.6\%) relapsed, of whom 23 (92\%) developed local relapses. The median time to the development of relapse was 7.6 months (range, 0.2-48.9 months). Nineteen relapses (76\%) were detected based on symptoms, physical examination and laboratory findings, and two relapses $(8 \%)$ were detected by radiological tests within the $1^{\text {st }}$ year after therapy. Only four patients $(16 \%)$ had their recurrence detected by surveillance imaging after the $1^{\text {st }}$ year. All the six patients who died relapsed within the $1^{\text {st }}$ year after therapy. No patient with a recurrence after 1 year has died, regardless of how the recurrence was diagnosed. The study concluded that detecting late HL relapse, whether by imaging or clinical change, did not affect OS. ${ }^{[50,69]}$

\section{Long-term Side Effects}

\section{Cardiac}

Anthracyclines (ATC) is a group of cytotoxic drugs that are commonly used for treatment of HL. However, the development of irreversible cardiotoxicity has prompted trials of use of combinations without the inclusion of ATCs. ATC use is limited by unique cumulative dose-limiting cardiotoxicity of approximately a total of $350 \mathrm{mg} / \mathrm{m}^{2}$. Overt heart failure (HF) occurs in nearly $4.5 \%-7 \%$ of all patients treated with ATC's. Patients may remain symptom free for several years. However, the prognosis becomes very poor after the development of frank HF. ATC-induced congestive HF is usually due to permanent changes in the myocardial muscles. Although many factors are proposed as possible causes, a large body of evidence points to $\mathrm{O}_{2}$ free-radical-mediated myocyte damage. The risk of developing HF is modified by the presence of certain risk factors that reduce cardiac tolerance to ATC's. Age and female gender seem to have higher incidence of ATC-induced cardiotoxicity. ATC cardiotoxicity can be divided, into acute, subacute, and a progressive late, 
chronic forms. Various methods were used to measure the extent of cardiac damage (invasive and noninvasive techniques). Due to the successful action of ATC's as CTR agents, several strategies have been tried to prevent or attenuate their side effects such as finding alternative CTR schedules, introducing special carriers of ATC's and using cardioprotective agents. ${ }^{[70,71]}$

Mediastinal RT seems to result in various degrees of cardiac valvular disease. In a cross-sectional study reported by Bijl et al., $82 \mathrm{HL}$ survivors were reviewed (52\% men, $48 \%$ women, mean age 47.8 years), fifty patients received mediastinal RT. Valvular disease was diagnosed by transthoracic echocardiography. During a median follow-up of 13.4 years (range 2-39 years), $\geq$ mild valvular disease was present in $61.2 \%$ of HL survivors who received mediastinal RT $(n=30)$, compared with $31.0 \%$ of HL survivors without mediastinal RT. Aortic valve regurgitation (AR) was most prevalent and patients who received mediastinal $\mathrm{RT}$ had significantly $\geq$ mild AR $(38.2 \%$ vs. $6.8 \%, P=0.007)$. Severe valvular disease was present in $24.5 \%$ of survivors with mediastinal RT compared with $3.4 \%$ without mediastinal RT $(P=0.016)$. Valvular surgery was performed in 9 survivors $(18.0 \%)$ with mediastinal RT and in none of the survivors without RT. The study concluded that the prevalence of cardiac valvular disease in HL survivors treated with mediastinal RT was high and seems to increase with time. ${ }^{[72]}$

Amini et al. conducted a study to evaluate the risk of cardiac death in pediatric HL survivors. The SEER program database was queried to analyze the rates of RT use and cardiac-specific mortality (CSM) in HL patients, aged $<21$ years, treated from 1973 to 2007 . The primary endpoint was cardiac mortality. A total of 6552 patients were included for a median follow-up of 12 years (range, 0-40). The median patient age at diagnosis was 17 years (range, 0-21 years). Majority of patients were white $(85.5 \%)$, from western states (41.2\%), had NS HL (73.2\%), presented with Stage I or II disease $(51.5 \%)$, and received RT $(56.1 \%)$. Death as a result of cardiac disease occurred in 114 patients $(9.2 \%)$. CSM for the whole cohort at 10, 20, and 30 - years were $0.3 \%, 1.6 \%$, and $5.0 \%$, respectively. The median age at the time of cardiac death was 39 years (range, 18-58 years). Adolescent patients (ages 13-21) had higher CSM rates (hazard ratio [HR], 3.05; $P=0.005$ ) under multivariate analysis (MVA). Female gender, patients treated from 1998 to 2007, and those with LR histology had significantly lower rates of CSM. Under MVA, use of RT was not associated with increased CSM. Male and adolescent patients at diagnosis were more likely to die of a cardiac-related causes. ${ }^{[73]}$

\section{Pulmonary}

Adolescents and young adults (AYA) treated for HL are at risk for lung function abnormalities, significantly more frequent in patients who received more intense treatment, such as mediastinal RT at a higher dose ( $>20 \mathrm{~Gy})$ and more CTR blocks. Pneumonitis and progressive fibrosis may follow treatment with carmustine, BCN, and RT. Few data is available about pulmonary complications in patients treated for HL in childhood or adolescent years. In a cross-sectional study carried out by Bossi et al., they evaluated the lung function abnormalities and respiratory symptoms in 27 patients (16 males and 11 females) diagnosed with HL between 1983 and 1994 (median age 11 years, range 2-16 years). All patients had been treated with CTR and RT according to the European protocols AIEOP-MH $83(n=14)$ or AIEOP-MH $89(n=13)$. Patients were followed up for a median time of 76 months after treatment completion. At the time of the study, 26 patients were in first $\mathrm{CR}$ and one in second $\mathrm{CR}$. Of the 27 patients, 19 had received mediastinal RT at a dose of 20 Gy $(n=5)$ or $20.8-44$ Gy $(n=14)$. Forced vital capacity (FVC), functional residual capacity, forced expiratory volume in one second (FEV1), FEV1/FVC ratio, and maximal expiratory flow at $25 \%$ of FVC were recorded and diffusion capacity for carbon monoxide (DLCO) was determined. Data were expressed as standard deviation (SD) score. Four patterns of respiratory function abnormalities were detected: restrictive, obstructive, isolated bronchiolar, and isolated diffusion impairment. Twelve patients (44\%) were asymptomatic and showed completely normal pulmonary function tests. Three patients suffered dyspnea on exertion, and one of them had productive cough. Out of these symptomatic subjects, only 1 patient had functional abnormality (isolated DLCO impairment). A restrictive pattern was found in 5 patients $(18 \%), 2$ of them also had a pathological DLCO SD score. Eight additional patients $(30 \%)$ had isolated diffusion impairment. The oxygen saturation was normal in all patients. Forty-seven percent of patients with normal DLCO received lower dose RT (20 Gy) compared to $10 \%$ of patients with impaired DLCO $(P=0.054)$. Similarly, patients with normal DLCO received significantly less CTR compared to patients with abnormal DLCO $(P=0.003)$. The development of lung abnormalities was not significantly associated with sex, age at treatment, mediastinal irradiation, and time elapsed since treatment completion. ${ }^{[74,75]}$

\section{Endocrine}

The intensity of chemo-RT combinations used to treat pediatric HL patients are associated with many long-term endocrine complications. Van Dorp et al. published a review article on long-term endocrine complications in survivors of childhood HL. He identified 16 studies (10 studies: 298 male survivors and 6 studies: 230 female survivors) on gonadal dysfunction. Survivors treated with alkylating agents or pelvic RT often developed severe gonadal damage and recovery was rarely the case. Gonadal dysfunction seems to be the most severe endocrine long-term complication, especially after treatment with alkylating CTR agents or pelvic RT. Seven studies (481 survivors) on 
bone mineral density (BMD) and growth were identified. The effects on BMD appear to be small. Data on growth are scarce but RT in a dose of $>30$ Gy including the spine, especially in prepubertal children, results in reduced final height. Ten studies (4012 survivors) on thyroid complications were also included. Hypothyroidism is the most common thyroid disorder after exposure to neck RT. There is also significant incidence of thyroid carcinoma developing after exposure to low-dose RT. Hypothyroidism and thyroid cancer have not been reported in survivors treated with CTR only. ${ }^{[76]}$

\section{Thyroid function}

Posttreatment thyroid disturbances are common in HL survivors. Patients, particularly those who received RT to the neck, must be closely followed up for occurrence of thyroid dysfunctions. The thyroid functions of 55 long-term survivors (M/F:2.05/1) of pediatric HL treated with CTR and RT was evaluated by Demirkaya et al. The mean age at diagnosis was $10.35 \pm 4.09$ (range: 2.83-17) years, and the mean follow-up period was $5.54 \pm 3.68$ (range: 0.92-13.92) years. All patients received CTR; a total of 50 patients (91\%) underwent RT, 42 (76.4\%) of whom received neck/mantle RT. Thyroid function tests were abnormal in $14(24.5 \%)$ patients. A diagnosis of subclinical and overt hypothyroidism was made in 11 (78.6\%) and $3(21.4 \%)$ respectively. Nearly one-fourth $(21.4 \%)$ of all thyroid function disorders were detected in the $1^{\text {st }}$ year of follow-up. significant correlation was found between the dose of mantle RT and abnormal thyroid functions $(P=0.002)$. In addition, statistically significant correlations were established between thyroid clinical examination, thyroid ultrasonography findings and thyroid function tests $\left(P<0.001\right.$ and $P=0.006$, respectively). ${ }^{[77]}$

\section{Gonadal function}

Gonadal toxicity, following successful treatment of $\mathrm{HL}$ in childhood using CMT and/or RT, has become an important problem. Krawczuk-Rybak et al. assessed the effect of CTR and RT on gonadal function in young male survivors after the treatment for HL. Levels of inhibin B, testosterone, follicle-stimulating hormone (FSH), luteinizing hormone (LH), and testicular volume were measured and assessed in $26 \mathrm{HL}$ survivors aged from 15.6 to 25.2 years, treated for HL during prepubertal $(n=8)$ or pubertal $(n=18)$ periods. In all groups, comparing to a control one, they found: (1) higher FSH concentration $(15.2 \pm 12.3 \mathrm{IU} / 1$ vs. $3.3 \pm 1.2 \mathrm{IU} / \mathrm{l})$; $P=0.0004$, lower inhibin B $(60.9 \pm 44.5 \mathrm{ng} / \mathrm{l} \mathrm{vs}$. $198.1 \pm 58.1 \mathrm{ng} / \mathrm{l}) ; \quad P=0.0001$, lower testicular volume $(18.2 \pm 2.6 \mathrm{ml}$ vs. $21.3 \pm 5.1 \mathrm{ml}) P=0.01$ and normal $\mathrm{LH}$ as well as testosterone values; (2) higher (>+2SD) FSH and $\mathrm{LH}$ were found in $62 \%$ and $23 \%$, respectively, and lower (<-2SD) inhibin B in $72 \%$ of survivors; (3) there were no differences between the patients: (a) treated before and during puberty and (b) patients with different stages of disease; (4) persistently lower inhibin B concentration and higher, but gradually normalized values of FSH were found $>6$ years after the end of therapy, (5) azoospermia was observed in 4/10 patients, oligospermia-in $4 / 10$ and normospermia-only in $2 / 10$ patients. In conclusion, CMT for HL, independently of the age and clinical stage, can lead to serious and persistent gonadal dysfunction. As the study indicated, patients should be counseled about gonadal toxicity and possibility of fertility preservation before starting therapy. ${ }^{[78]}$

De Bruin et al. conducted a cohort study among 518 females $\geq 5$-year HL survivors, aged 14-40 (median: 25) years at treatment (1965-1995). Multivariable Cox-regression was used to determine the treatment effects on the risk of development of premature menopause, defined as cessation of menses before age 40 years. After a median follow-up period of 9.4 years, 97 women entered menopause before age 40 years. Use of CTR was associated with a 12.3-fold increased risk of developing premature menopause compared with RT alone. Treatment with $\mathrm{MOPP} / \mathrm{ABV}$ combination significantly increased the risk of premature menopause (HR: 2.9). The alkylating agents PZN (HR: 8.1) and CPA (HR: 3.5), showed the strongest associations. After high cumulative doses $\left(>8.4 \mathrm{~g} / \mathrm{m}^{2}\right)$ of PZN, the actuarial risk of premature menopause was $64 \%$ at 10 years after treatment. The cumulative risk of premature menopause (at age $\leq 40$ years) did not significantly differ according to age, but time to premature menopause was much longer in women treated at early ages. As long as alkylating agents will continue to be used for treatment of HL, premature menopause will remain a significant devastating adverse treatment long-term complication. ${ }^{[79]}$

\section{Risk of stroke}

The Childhood Cancer Survivor Study (multi-institutional cohort study) reported the incidence of stroke in long term ( $>5$-years) HL survivors diagnosed between 1970 and 1986 compared to a sibling group. The incidence of stroke among survivors of HL $(n=1.926)$ and siblings $(n=3.846)$ were compared. The relative risk (RR) of developing stroke was calculated using Cox proportional hazards models. Nine siblings developed strokes, for an incidence of 8.00 per 100,000 person-years (95\% CI, 3.85-14.43 per 100,000 person-years). Twenty-four HL survivors reported a stroke. The incidence of late-occurring stroke among survivors of HL treatment was 83.6 per 100,000 person-years $(95 \%$ CI, 54.5-121.7 per 100,000 person-years). The RR of stroke among survivors of HD was 4.32 (95\% CI, 2.01-9.29; $P=0.0002)$. All 24 long term survivors received mantle RT (median dose, $40 \mathrm{~Gy}$ ). The incidence of late-stroke among survivors of HL treatment who received mantle RT was 109.8 per 100,000 person-years $(95 \%$ CI, $70.8-161.1$ per 100,000 person-years) with a RR of 5.62 (95\% CI, 2.59-12.25; $P<0.0001)$. Survivors of childhood HL are at increased risk of late stroke. Exposure to Mantle RT 
is strongly associated with the development stroke. The potential mechanisms implicated may include carotid artery or cardiac valvular disease. ${ }^{[80]}$

\section{Risk of second malignant neoplasms}

Survivors of HL treatment remain at risk of developing SMN's including leukemia, sarcomas, breast, thyroid, gastrointestinal, skin, and lung cancers. While early secondary leukemia is more associated with alkylating agents and epipodophyllotoxins, the risk of secondary solid tumors is late and is more closely linked to RT exposure, particularly at higher doses. Over the past 40 years, treatment of childhood HL has evolved from high-dose extended-field RT to CMT with CTR and low-dose IFRT. Such treatment strategies have the theoretical benefit of reduced risk of solid SMN due to decreased exposure to RT. Early reports of low incidence of SMN in children and young adults after low-dose RT are promising but confounded by the short follow-up (median, 8-13 years) periods. In 1970, and in an effort to diminish devastating effects of high-dose RT on growth and skeletal development in children, Stanford investigators pioneered a CMT protocol with MOPP CTR and low-dose IFRT. Children treated according to this protocol had normal growth, but many patients developed secondary leukemias and male infertility. In response, a second protocol was initiated in 1982 combining alternating cycles of MOPP and ABVD and consolidation low-dose IFRT. The median follow-up for patients treated on these protocols is now $>25$ years, allowing for the first long-term follow-up reports of pediatric HL survivors treated with CTR and low-dose IFRT. ${ }^{[39,81]}$

Dörffel et al. reported the outcomes of the long-term follow-up of 2548 pediatric HL patients treated in Europe between 1978 and 2002. More than 90\% of patients survived for 20 years or more. A total of 147 cases of SMN were diagnosed in 138/2548 patients (5.4\%), $47(32 \%)$ thyroid cancer, 37 (25\%) breast cancer, and $15(10 \%)$ hematopoietic malignancies. The cumulative incidence of SMN at 20, 25, and 30 years was $7 \%, 11.2 \%$, and $18.7 \%$, respectively. These reported percentages are rather low compared to other international studies. Among the 123 patients (84\%) with secondary solid tumors, $105(85 \%)$ had a tumor within the RT field. ${ }^{[82]}$ Women treated for HL in childhood or adolescence have an increased risk of developing breast cancer as young adults. The risk is much increased in patients treated with RT at younger age. Schellong et al., reported the results of the German HL studies on the incidence and occurrence of secondary breast cancer (sBC) in women treated for HL in childhood using CTR and RT. The study included 590 women treated in five consecutive pediatric HL trials between 1978 and 1995. Patients were then re-evaluated in a late follow-up study after a median interval of 17.8 years. By July 2012, sBC had been diagnosed in 26 of 590 females. The breast cancer occurred in the RT field in 25 of these 26 patients.
sBC was discovered with a median latency of 20.7 years after treatment (shortest latency, 14.3 years) and at a median age of 35.3 years (youngest age, 26.8 years). The RT dose to the supradiaphragmatic fields ranged from 20 to $45 \mathrm{~Gy}$. The cumulative incidence for $\mathrm{sBC}$ at 30 years after treatment was $19 \%(95 \% \mathrm{CI}, 12 \%-29 \%)$. For women aged between 25 and 45 years, the frequency of sBC was 24 times higher than the normal population. ${ }^{[83]}$

\section{Future Prospects for Pediatric, Adolescent and Young Adult Patients}

Pediatric HL is a potentially highly curable malignancy, however, long-term side effects of therapy need to be considered in optimizing clinical care. An expert panel was convened to reach consensus on the most appropriate method to evaluate and treatment of pediatric HL. The American College of Radiology Appropriateness Criteria are evidence-based guidelines for specific clinical conditions in pediatric HL that are reviewed every 2 years by a multidisciplinary expert panel. The guideline development includes an extensive review and analysis of the recent medical literature from highly ranked peer reviewed journals and the application of consensus methodology (modified Delphi method) to ensure the appropriateness of imaging and treatment procedures. In case evidence is lacking, or not definitive, expert opinion may be used to recommend imaging or treatment strategy. By combining available data and expert medical opinion, a consensus to the modern approach for the appropriate management of pediatric HL was provided. ${ }^{[84]}$

The outcome of treatment in AYA age $>15-<21$ years were compared to children treated for $\mathrm{HL}$ in two nonrandomized COG studies (P9425 and P9426) that used dose-intense, response-based CTR and a reduced dose IFRT. Patients $\leq 21$ years were eligible. Individuals with low-risk HL (stages IA, IIA, and IIIA1) without large mediastinal adenopathy, eligible for P9426, were treated with 2-4, 4 weekly cycles of ABVE and IFRT (25.5 Gy). Subjects with intermediate-risk HL (stages IB, IIA, IIIA1 with large mediastinal adenopathy, and IIIA2) and high-risk (stages IIB, IIIB, and IV), eligible for P9425, were treated with 3-5, 3 weekly cycles of ABVE-PC and IFRT (21 Gy). The 5-year EFS was compared in children versus that of AYA. Four hundred and seventy-one patients were enrolled on P9425 and P9426 studies. Of these, 203 were AYA and 104 with intermediate or high-risk, and 99 with low-risk HL. With a median follow-up of 7.7 years, the 5-year EFS of children did not significantly differ from that of AYA (85.9 vs. $87.1 \%, P=0.51)$. The study concluded that AYA have good outcomes when treated according to pediatric protocols. Given the equivalent and excellent results of therapy, HL represents an opportunity for adult and pediatric study collaborative groups to design clinical trials targeted to AYA focusing on treatment efficacy in addition to the quality of life of AYA and in reduction of long-term side effects. ${ }^{[85]}$ 
Upfront treatment of pediatric HL has reached great success in curing patients using conventional CMT. Refinement of treatment is being currently undertaken in several trials to try and further reduce CTR based on response and hence reduce toxicity. Treatment of relapse/refractory HL still gives no optimal results. The use of conventional CTR and RT methods has probably reached the ceiling. Newer targeted medications and immunotherapy are probably the way to go. Recent advances in the understanding of the pathogenesis of classical HL; the interaction within the tumor microenvironment, and the tumor immune-escape mechanisms have led to discovery of novel therapeutic targets. The breakthrough came with the discovery of brentuximab vedotin, a monoclonal antibody targeting cluster of differentiation 30 of classic HL. BV was approved by the Food and Drug Administration in 2011 for treatment of adult and pediatric patients with relapsed/refractory classical HL with good success and reduced toxicity. ${ }^{[65]}$

Pembrolizumab is another humanized monoclonal antibody targeting programmed cell death protein 1 (PD-1) receptors, a key CD4+ T-cell inhibitory molecule directly involved in tumor T-cell immune-escape mechanisms. PD-1 ligands are upregulated on HRS cells. This is thought to be initiated by chromosome 9p24.1 amplification and EB virus infection. Pembrolizumab and its partner drug nivolumab have shown significant activity and high response rate in adult patients with relapsed/refractory HL and acceptable toxicity. Trials in children are underway and given the similarities between pediatric and adult HL, pediatric investigators are optimistic to achieve similarly good results..$^{[86,87]}$

\section{Declaration of patient consent}

The authors certify that they have obtained all appropriate patient consent forms. In the form the patient(s) has/have given his/her/their consent for his/her/their images and other clinical information to be reported in the journal. The patients understand that their names and initials will not be published and due efforts will be made to conceal their identity, but anonymity cannot be guaranteed.

\section{Acknowledgment}

The authors would like to thank Dr. Aieshah Alazemi for her valuable contribution in preparing the illustrations for publication.

\section{Financial support and sponsorship}

Nil.

\section{Conflicts of interest}

There are no conflicts of interest.

\section{References}

1. Longo DL, Armitage JO. Controversies in the treatment of early-stage Hodgkin's lymphoma. N Engl J Med 2015;372:1667-9.

2. Surveillance, Epidemiology, and End Results (SEER) Program
Data 2017. USA: National Cancer Institute (NCI). Available from: https://seer.cancer.gov/. [Last accessed on 2019 Sep 20].

3. Medeiros LJ, Greiner TC. Hodgkin's disease. Cancer 1995;75:357-69.

4. Schwartz CL. Special issues in pediatric Hodgkin's disease. Eur J Haematol Suppl 2005;(66):55-62.

5. Wiegner EA, Donaldson SS. Controversies in radiotherapy for pediatric Hodgkin's lymphoma. Expert Rev Anticancer Ther 2011;11:1357-66.

6. Mendenhall NP, Cantor AB, Williams JL, Ternberg JL, Weiner MA, Kung FH, et al. With modern imaging techniques, is staging laparotomy necessary in pediatric Hodgkin's diseases? A Pediatric Oncology Group study. J Clin Oncol 1993;11:2218-25.

7. Swerdlow SH, Campo E, Harris NL, Jaffe ES, Pileri S, Stein H. WHO Classification of tumors of hematopoietic and lymphoid tissue. International agency for Research on Cancer, Lyon. (4 ${ }^{\text {th }}$ ed.) 2008. Blood 2011;117:5019-32.

8. Hansmann ML, Willenbrock K. WHO classification of Hodgkin's lymphoma and its molecular pathological relevance. Pathologe 2002;23:207-18.

9. Huppmann AR, Nicolae A, Slack GW, Pittaluga S, Davies-Hill T, Ferry JA, et al. EBV may be expressed in the LP cells of nodular lymphocyte-predominant Hodgkin lymphoma (NLPHL) in both children and adults. Am J Surg Pathol 2014;38:316-24.

10. Grewal R, Irimie A, Naidoo N, Mohamed N, Petrushev B, Chetty M, et al. Hodgkin's lymphoma and its association with EBV and HIV infection. Crit Rev Clin Lab Sci 2018;55:102-14.

11. Jarrett RF. Risk factors for Hodgkin's lymphoma by EBV status and significance of detection of EBV genomes in serum of patients with EBV-associated Hodgkin's lymphoma. Leuk Lymphoma 2003;44 Suppl 3:S27-32.

12. Meyer RM. EBV DNA: A Hodgkin lymphoma biomarker? Blood 2013;121:3541-2.

13. Park JH, Yoon DH, Kim S, Park JS, Park CS, Sung H, et al. Pretreatment whole blood Epstein -Barr virus - DNA is a significant prognostic marker in patients with Hodgkin's lymphoma. Ann Hematol 2016;95:801-8.

14. Kirkorian JG, Portlock CS, Mauch PM. Hodgkin's disease presenting below the diaphragm: A review. J Clin Oncol 1986;4:1551-62.

15. Li ZM, Zhu YJ, Xia Y, Huang JJ, Jiang WQ. Clinical characteristics of the patients with Hodgkin's lymphoma involving extranodal sites. Chin J Cancer 2012;31:342-7.

16. Hudson MM, Schwartz C, Constine LS. Treatment of pediatric Hodgkin's lymphoma. In: Weinstein HJ, Hudson MM, Link MP, editors. Pediatric Lymphomas, First edition, Springer - Verlag Publishers, Berlin, Heidelberg, Germany; 2007. p. 423.

17. Peng Y, Qing AC, Cai J, Yue C, French SW, Qing X. Lymphoma of the liver: Clinicopathological features of 19 patients. Exp Mol Pathol 2016;100:276-80.

18. Padhan RK, Das P, Shalimar. Primary hepatic lymphoma. Trop Gastroenterol 2015;36:14-20.

19. Dich NH, Goodman ZD, Klein MA. Hepatic involvement in Hodgkin's disease. Clues to histologic diagnosis. Cancer 1989;64:2121-6.

20. Binesh F, Halvani H, Taghipour S, Navabii H. Primary pulmonary classic Hodgkin's lymphoma. BMJ Case Rep 2011;2011. pii: bcr0320113955.

21. Kolygin BA, Vesnin AG. Hodgkin's disease in children: Involvement of lung and pleura. Pediatr Radiol 1976;5:75-80.

22. Ha-ou-Nou FZ, Benjilali L, Essaadouni L. Sacral pain as the initial symptom in primary Hodgkin's lymphoma of bone. J Cancer Res Ther 2013;9:511-3. 
23. Singh P, Bakhshi S. Osseous involvement in pediatric Hodgkin's lymphoma. Indian J Pediatr 2010;77:565-6.

24. Martinez DL, Gujrati M, Geoffroy F, Tsung AJ. Isolated CNS Hodgkin's lymphoma: Implications for tissue diagnosis. CNS Oncol 2014;3:383-7.

25. Gessi M, Kuchelmeister K, Kellner U, Ritter M, Morgner A, Urbach $\mathrm{H}$, et al. Unusual clinico-pathological features in primary Hodgkin's lymphomas of the central nervous system. Acta Neurochir (Wien) 2013;155:19-24.

26. van Blydenstein SA, Patel M, Philip V, Lakha A, Pather S, Westgarth-Taylor $\mathrm{T}$, et al. Classical Hodgkin Lymphoma involving the central nervous system (brain) - An unusual presentation. Clin Case Rep 2014;2:88-92.

27. Tassies D, Sierra J, Montserrat E, Martí R, Estrach T, Rozman C. Specific cutaneous involvement in Hodgkin's disease. Hematol Oncol 1992;10:75-9.

28. Carbone PP, Kaplan HS, Musshoff K, Smithers DW, Tubiana M. Report of the committee on Hodgkin's disease staging classification. Cancer Res 1971;31:1860-1.

29. Afaq A, Fraioli F, Sidhu H, Wan S, Punwani S, Chen SH, et al. Comparison of PET/MRI with PET/CT in the evaluation of disease status in lymphoma. Clin Nucl Med 2017;42:e1-7.

30. Subocz E, Hałka J, Dziuk M. The role of FDG-PET in Hodgkin lymphoma. Contemp Oncol (Pozn) 2017;21:104-14.

31. Freed J, Kelly KM. Current approaches to the management of pediatric Hodgkin lymphoma. Paediatr Drugs 2010;12:85-98.

32. Castellani MR, Cefalo G, Terenziani M, Aliberti G, Maccauro M, Alessi A, et al. Gallium scan in adolescents and children with Hodgkin's disease (HD). Treatment response assessment and prognostic value. Q J Nucl Med 2003;47:22-30.

33. Escobar GI, Lopez A, Rubio J, Perez-Callejo D, Barrigón DC, Alonso PT, et al. Clinical pretreatment risk factors and prediction of outcome using gallium 67 scintigraphy in patients with Hodgkin's lymphoma. Mol Clin Oncol 2016;4:93-9.

34. Cole PD, McCarten KM, Drachtman RA, De Alarcon P, Chen L, Trippett TM, et al. Early FDG-PET-based response evaluation after treatment with gemcitabine and vinorelbine for refractory Hodgkin disease: A Children's Oncology Group Report. Pediatr Hematol Oncol 2010;27:650-7.

35. Lin HM Teitell MA. Second malignancy after treatment of pediatric Hodgkin's lymphoma. J Pediatr Hematol Oncol 2005;27:28-36.

36. Lipshultz SE, Colan SD, Gelber RD, Perez-Atayde AR, Sallan SE, Sanders SP. Late cardiac effects of doxorubicin therapy for acute lymphoblastic leukemia in childhood. N Engl J Med 1991;324:808-15.

37. Marina VM, Greenwald CA, Fairclough DL, Thompson EI, Wilimas JA, Mackert PW, et al. Serial pulmonary function studies in children treated for newly diagnosed Hodgkin's disease with mantle radiotherapy plus cycles of cyclophosphamide, vincristine and procarbazine alternating with cycles of doxorubicin, bleomycin, vinblastine and dacarbazine. Cancer 1995;75:1706-11.

38. Canellos GP. Can MOPP be replaced in the treatment of advanced Hodgkin's disease? Semin Oncol 1990;17:2-6.

39. van den Berg H, Stuve W, Behrendt H. Treatment of Hodgkin's disease in children with alternating mechlorethamine, vincristine, procarbazine, and prednisone (MOPP) and adriamycin, bleomycin, vinblastine, and dacarbazine (ABVD) courses without radiotherapy. Med Pediatr Oncol 1997;29:23-7.

40. Hay AE, Meyer RM. Balancing risks and benefits of therapy for patients with favorable-risk limited-stage Hodgkin lymphoma: The role of ABVD chemotherapy alone. Hematol Oncol Clin North Am 2014;28:49-63.
41. Probert JC, Parker BR, Kaplan HS. Growth retardation in children after megavoltage irradiation of the spine. Cancer 1973;32:634-9.

42. Donaldson SS, Link MP. Combined modality treatment with low dose radiation and MOPP chemotherapy for children with Hodgkin's disease. J Clin Oncol 1987;5:742-9.

43. Specht L. Prognostic factors in Hodgkin's disease. Semin Radiat Oncol 1996;6:146-61.

44. Moccia AA, Donaldson J, Chhanabhai M, Hoskins PJ, Klasa RJ, Savage KJ, et al. International Prognostic Score in advanced-stage Hodgkin's lymphoma: Altered utility in the modern era. J Clin Oncol 2012;30:3383-8.

45. Schwartz CL, Chen L, McCarten K, Wolden S, Constine LS, Hutchison RE, et al. Childhood Hodgkin's International Prognostic Score (CHIPS) Predicts eventfree survival in Hodgkin's Lymphoma: A Report from the Children's Oncology Group. Pediatr Blood Cancer 2017;64:1-18 [doi: 10.1002/pbc. 26278].

46. Giulino-Roth L, Keller FG, Hodgson DC, Kelly KM. Current approaches in the management of low risk Hodgkin lymphoma in children and adolescents. Br J Haematol 2015;169:647-60.

47. Dörffel W, Rühl U, Lüders $H$, Claviez A, Albrecht $M$, Bökkerink J, et al. Treatment of children and adolescents with Hodgkin lymphoma without radiotherapy for patients in complete remission after chemotherapy: Final results of the multinational trial GPOH-HD95. J Clin Oncol 2013;31:1562-8.

48. Radford J, Illidge T, Counsell N, Hancock B, Pettengell R, Johnson $\mathrm{P}$, et al. Results of a trial of PET-directed therapy for early - Stage Hodgkin's lymphoma. N Engl J Med 2015;372:1598-607.

49. Donaldson SS, Link MP, Weinstein HJ, Rai SN. Final results of a prospective clinical trial with VAMP and low-dose involved-field radiation for children with low-risk Hodgkin's disease. J Clin Oncol 2007;25:332-7.

50. Friedman DL, Chen L, Wolden S, Buxton A, McCarten K, FitzGerald TJ, et al. Dose-intensive response-based chemotherapy and radiation therapy for children and adolescents with newly diagnosed intermediate-risk Hodgkin lymphoma: A Report from the Children's Oncology Group Study AHOD0031. J Clin Oncol 2014;32:3651-8

51. Hehn ST, Miller TP. What is the treatment of choice for advanced-stage Hodgkin's lymphoma: ABVD, Stanford V, or BEACOPP? Curr Hematol Rep 2004;3:17-26.

52. Viviani S, Zinzani PL, Rambaldi A, Brusamolino E, Levis A, Bonfante $\mathrm{V}$, et al. ABVD versus BEACOPP for Hodgkin's lymphoma when high-dose salvage is planned. N Engl J Med 2011;365:203-12.

53. Kelly KM, Sposto R, Hutchinson R, Massey V, McCarten K, Perkins $\mathrm{S}$, et al. BEACOPP chemotherapy is a highly effective regimen in children and adolescents with high-risk Hodgkin lymphoma: A report from the Children's Oncology Group. Blood 2011;117:2596-603.

54. Schwartz CL, Constine LS, Villaluna D, London WB, Hutchison RE, Sposto R, et al. A risk-adapted, response-based approach using ABVE-PC for children and adolescents with intermediate- and high-risk Hodgkin lymphoma: The results of P9425. Blood 2009;114:2051-9.

55. Savage KJ, Mottok A, Fanale M. Nodular lymphocyte-predominant Hodgkin lymphoma. Semin Hematol 2016;53:190-202.

56. Gorde-Grosjean S, Guimard G, Lambilliotte A, Coulomb-Lhermine A, Montravers F, Landman-Parker J. Nodular lymphocyte-predominant Hodgkin lymphoma in 
children: Clinical course, biology, and management. Bull Cancer 2014;101:881-90.

57. van Grotel M, Lam KH, de Man R, Beishuizen A, Pieters R, van den Heuvel-Eibrink MM. High relapse rate in children with non-advanced nodular lymphocyte predominant Hodgkin's lymphoma (NLPHL or nodular paragranuloma) treated with chemotherapy only. Leuk Lymphoma 2006;47:1504-10.

58. Schlembach PJ, Wilder RB, Jones D, Ha CS, Fayad LE, Younes A, Hagemeister F, et al. Radiotherapy alone for lymphocyte-predominant Hodgkin's disease. Cancer J 2002;8:377-83.

59. Claviez A, Sureda A, Schmitz N. Hematopoietic SCT for children and adolescents with relapsed and refractory Hodgkin's lymphoma. Bone Marrow Transplant 2008;42 Suppl 2:S16-24.

60. Akhtar S, Rauf SM, Elhassan TA, Maghfoor I. Outcome analysis of high-dose chemotherapy and autologous stem cell transplantation in adolescent and young adults with relapsed or refractory Hodgkin lymphoma. Ann Hematol 2016;95:1521-35.

61. Devillier R, Coso D, Castagna L, Brenot Rossi I, Anastasia A, Chiti A, et al. Positron emission tomography scan response at the time of autologous transplant predicts outcome of patients with relapse/refractory Hodgkin's lymphoma. Haematologica 2012;97:1073-9.

62. Cole PD, McCarten KM, Pei Q, Spira M, Metzger ML, Drachtman RA, et al. Brentuximab vedotin with gemcitabine for paediatric and young adult patients with relapsed or refractory Hodgkin's lymphoma (AHOD1221): A Children's Oncology Group, multicentre single-arm, phase 1-2 trial. Lancet Oncol 2018;19:1229-38.

63. Younes A. Brentuximab vedotin for the treatment of patients with Hodgkin lymphoma. Hematol Oncol Clin North Am 2014;28:27-32.

64. Bonthapally V, Wu E, Macalalad A, Yang H, Shonukan O, Liu Y, et al. Brentuximab vedotin in relapsed/refractory Hodgkin lymphoma post-autologous transplant: Meta-analysis versus historical data. Curr Med Res Opin 2015;31:993-1001.

65. Chen R, Palmer JM, Martin P, Tsai N, Kim Y, Chen BT, et al. Results of a multicenter phase II trial of brentuximab vedotin as second-line therapy before autologous transplantation in relapsed/refractory Hodgkin lymphoma. Biol Blood Marrow Transplant 2015;21:2136-40.

66. Gallamini A, Barrington SF, Biggi A, Chauvie S, Kostakoglu L, Gregianin $\mathrm{M}$, et al. The predictive role of interim positron emission tomography for Hodgkin lymphoma treatment outcome is confirmed using the interpretation criteria of the Deauville five-point scale. Haematologica 2014;99:1107-13.

67. Adams HJ, Kwee TC. Prognostic value of pre-transplant FDG-PET in refractory/relapsed Hodgkin's lymphoma treated with autologous stem cell transplantation: Systematic review and meta-analysis. Ann Hematol 2016;95:695-706.

68. Mauz-Körholz C, Metzger ML, Kelly KM. Pediatric Hodgkin lymphoma. J Clin Oncol 2015;33:2975-85.

69. Voss SD, Chen L, Constine LS, Chauvenet A. Surveillance computed tomography imaging and detection of relapse in intermediate- and advanced-stage pediatric Hodgkin's lymphoma: A report from the Children's Oncology Group. J Clin Oncol 2012;30:2635-40.

70. Ferreira AL, Matsubara LS, Matsubara BB. Anthracycline induced cardiotoxicity. Cardiovasc Hematol Agents Med Chem 2008;6:278-81.

71. Larussi $\mathrm{D}$, Indolfi $\mathrm{P}$, Galderisi $\mathrm{M}$, Bossone $\mathrm{E}$. Cardiac toxicity after anthracycline chemotherapy in childhood. Herz 2000;25:676-88.
72. Bijl JM, Roos MM, van Leeuwen-Segarceanu EM, Vos JM, Bos WW, Biesma DH, et al. Assessment of valvular disorders in survivors of Hodgkin's lymphoma treated by mediastinal radiotherapy \pm chemotherapy. Am J Cardiol 2016;117:691-6.

73. Amini A, Murphy B, Cost CR, Garrington TP, Greffe BS, Liu AK. Cardiac mortality in children and adolescents with Hodgkin's lymphoma: A surveillance, epidemiology and end results analysis. J Adolesc Young Adult Oncol 2016;5:181-6.

74. Bossi G, Cerveri I, Volpini E, Corsico A, Baio A, Corbella F, Klersy C, et al. Long-term pulmonary sequelae after treatment of childhood Hodgkin's disease. Ann Oncol 1997;8 Suppl 1:19-24.

75. Hohl RJ, Schilsky RL. Nonmalignant complications of therapy for Hodgkin's disease. Hematol Oncol Clin North Am 1989;3:331-43.

76. Van Dorp W, van Beek RD, Laven JS, Pieters R, de Muinck Keizer-Schrama SM, van den Heuvel-Eibrink MM, et al. Long-term endocrine side effects of childhood Hodgkin's lymphoma treatment: A review. Hum Reprod Update 2012;18:12-28.

77. Demirkaya M, Sevinir B, Sağlam H, Özkan L, Akacı O. Thyroid functions in long-term survivors of pediatric Hodgkin's lymphoma treated with chemotherapy and radiotherapy. J Clin Res Pediatr Endocrinol 2011;3:89-94.

78. Krawczuk-Rybak M, Płonowski M, Solarz E, Leszczyńska E, Wojtkowska M, Wysocka J, et al. Gonadal and thyroid function after treatment for Hodgkin's lymphoma in adolescents. Med Wieku Rozwoj 2008;12 (4 Pt 2):1014-20.

79. De Bruin ML, Huisbrink J, Hauptmann M, Kuenen MA, et al. Treatment-related risk factors for premature menopause following Hodgkin lymphoma. Blood 2008;111:101-8.

80. Bowers DC, McNeil DE, Liu Y, Yasui Y. Stroke as a late treatment effect of Hodgkin's disease: A report from the Childhood Cancer Survivor Study. J Clin Oncol 2005;23:6508-15.

81. O'Brien MM, Donaldson SS, Balise RR, Whittemore AS, Link MP. Second malignant neoplasms in survivors of pediatric Hodgkin's lymphoma treated with low-dose radiation and chemotherapy. J Clin Oncol 2010;28:1232-9.

82. Dörffel W, Riepenhausen $M$, Lüders $H$, Brämswig $J$, Schellong G. Secondary malignancies following treatment for Hodgkin's lymphoma in childhood and adolescence. A cohort study with more than 30 years' follow-up. Dtsch Arztebl Int 2015;112:320-7.

83. Schellong $\mathrm{G}$, Riepenhausen $\mathrm{M}$, Ehlert $\mathrm{K}$, Brämswig J, Schmutzler RK, Rhiem $\mathrm{K}$, et al. Breast cancer in young women after treatment for Hodgkin's disease during childhood or adolescence - An observational study with up to 33-year follow-up. Dtsch Arztebl Int 2014;111:3-9.

84. Terezakis SA, Metzger ML, Hodgson DC, Schwartz CL, Advani R, Flowers CR, Hoppe BS, et al. ACR appropriateness criteria pediatric Hodgkin lymphoma. Pediatr Blood Cancer 2014;61:1305-12.

85. Fernández KS, Schwartz CL, Chen L, Constine LS, Chauvenet A, de Alarcón PA, et al. Outcome of adolescents and young adults compared to children with Hodgkin lymphoma treated with response-based chemotherapy on pediatric protocols: A Children's Oncology Group report. Pediatr Blood Cancer 2017;64:10.1002/pbc.26681. Epub 2017 Jun 14.

86. Maly J, Alinari L. Pembrolizumab in classical Hodgkin's lymphoma. Eur J Haematol 2016;97:219-27.

87. Chen R, Zinzani PL, Fanale MA, Armand P, Johnson NA, Brice $\mathrm{P}$, et al. Phase II study of the efficacy and safety of pembrolizumab for relapsed/refractory classic Hodgkin lymphoma. J Clin Oncol 2017;35:2125-32. 